\title{
CONTRIBUTION \\ A LA BIOLOGIE DES ALGUES D'EAU DOUCE D'UN ETANG BRETON. I : LE MILIEU NATUREL
}

\author{
par B. SAVOuRÉ.
}

\section{SOMMAIRE}

Introduction $\ldots \ldots \ldots \ldots \ldots \ldots \ldots \ldots \ldots \ldots \ldots \ldots \ldots \ldots \ldots$

I. - Les stations étudiées $\ldots \ldots \ldots \ldots \ldots \ldots \ldots \ldots \ldots \ldots \ldots . \ldots \ldots$

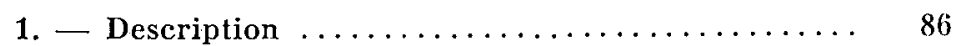

2. - Conditions climatiques $\ldots \ldots \ldots \ldots \ldots \ldots \ldots . \quad 89$

II. - Analyse physico-chimique des milieux $\ldots \ldots \ldots \ldots \ldots \ldots .69$

1. - La transparence des eaux ............ 90

2. - La température $\ldots \ldots \ldots \ldots \ldots \ldots \ldots \ldots \ldots .96 \ldots$

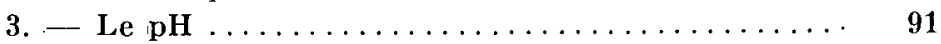

4. - L'oxygène dissous $\ldots \ldots \ldots \ldots \ldots \ldots \ldots \ldots \ldots \ldots . \ldots \ldots$

5. — Le gaz carbonique libre $\ldots \ldots \ldots \ldots \ldots \ldots \ldots \ldots . \ldots 2$

6. - L'alcalinité totale $\ldots \ldots \ldots \ldots \ldots \ldots \ldots \ldots \ldots . \ldots 2$

7. - Les anions et cations . . . . . . . . . 93

(7.1. - Les sulfates; 7.2. - Les nitrates; 7.3. Les chlorures; 7.4. - L'azote ammoniacal; 7.5. Les phosphates; 7.6. - Le calcium; 7.7. - Le magnésium; 7.8. - La silice; 7.9. - I.e fer; 7.10 Les oligo-éléments : cobalt, cuivre, molybdène).

III. - Rappel de la typologie des eaux douces bretonnes et typologie des eaux de l'étang étudié $\ldots \ldots \ldots \ldots \ldots \ldots \ldots \ldots \ldots \quad 99$

IV. - Variations saisonnières des facteurs physico-chimiques; traitement mathématique des données .............. 100

1. - La technique expérimentale ........... 100

2. - L'analyse proprement dite $\ldots \ldots \ldots \ldots \ldots \ldots \ldots .103$

3. - Les résultats $\ldots \ldots \ldots \ldots \ldots \ldots \ldots \ldots \ldots \ldots \ldots .103$

(3.1. - L'analyse globale; 3.2. - Décomposition de l'analyse globale; 3.3 . - Les analyses partielles).

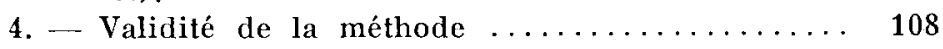

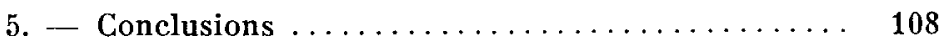

v. - Conclusions générales $\ldots \ldots \ldots \ldots \ldots \ldots \ldots \ldots \ldots \ldots \ldots \ldots$ 


\section{INTRODUCTION}

Nous tentons de préciser depuis quelques années l'intervention du milieu sur la composition et la dynamique saisonnière des populations algales d'un étang breton (étang de Planche-Roger).

Nous nous limitons dans cette première partie à définir notre milieu naturel. Ce premier travail est indispensable. En effet, si nous voulons comprendre l'influence des facteurs du milieu sur la croissance des algues en place, il est nécessaire de décrire avec précision les variations de ces facteurs au cours d'un cycle complet de végétation. Cependant il est difficile de manœuvrer toutes les données. Aussi avons-nous tenté d'exprimer les ressemblances et les différences entre facteurs du milieu et stations (les biotopes choisis étant définis par de nombreux caractères qu'ils possèdent en commun à des degrès variables). Le traitement mathématique choisi, dit des Correspondances et défini par BENzEch I et collaborateurs s'est révélé très prometteur en recherche médicale [BounEL : 1968]. Il consiste ici à représenter simultanément les facteurs physico-chimiques en respectant au mieux les données initiales.

\section{I. - LES STATIONS ÉTUDIEES}

\section{1. - Description.}

L'étang de Planche-Roger (fig. 1) situé sur l'axe routier Rennes Antrain-Pontorson près de la commune de Feins (Ille-et-Vilaine) est une importante réserve de chasse et de pêche appartenant au service national des Ponts et Chaussées. Aligné sur des alluvions modernes suivant une direction est-ouest, il est limité au nord par la forêt de la Fertais et au sud par une large zone de champs cultivés. Son plan d'eau peut atteindre trois hectares et la profondeur optimale ne dépasse pas trois mètres à la bonde. Un système de petites digues de terre battue permet de retenir à tout moment les eaux du ruisseau d'alimentation dit du Bourguet. Ces eaux peuvent être canalisées le long de la rive nord jusqu'au grand réservoir de pisciculture du Boulet. Cet étang peut donc être mis à sec très rapidement; cette opération a lieu chaque hiver en décembre. Les berges de cet étang interdites aux promeneurs ne subissent donc jamais l'influence du facteur anthropobiotique. Par contre, l'action du facteur anthronozoobiotique est importante. En effet, des troupeaux de bovidés vont s'abreuver assez souvent en période estivale et automnale le long de la rive sud.

Les associations végétales aquatiques décrites avec précision par LENOIR [1958] en Bretagne sont bien représentées. La zone centrale est composée d'un Myriophylletum dont la caractéristique est $M y$ - 


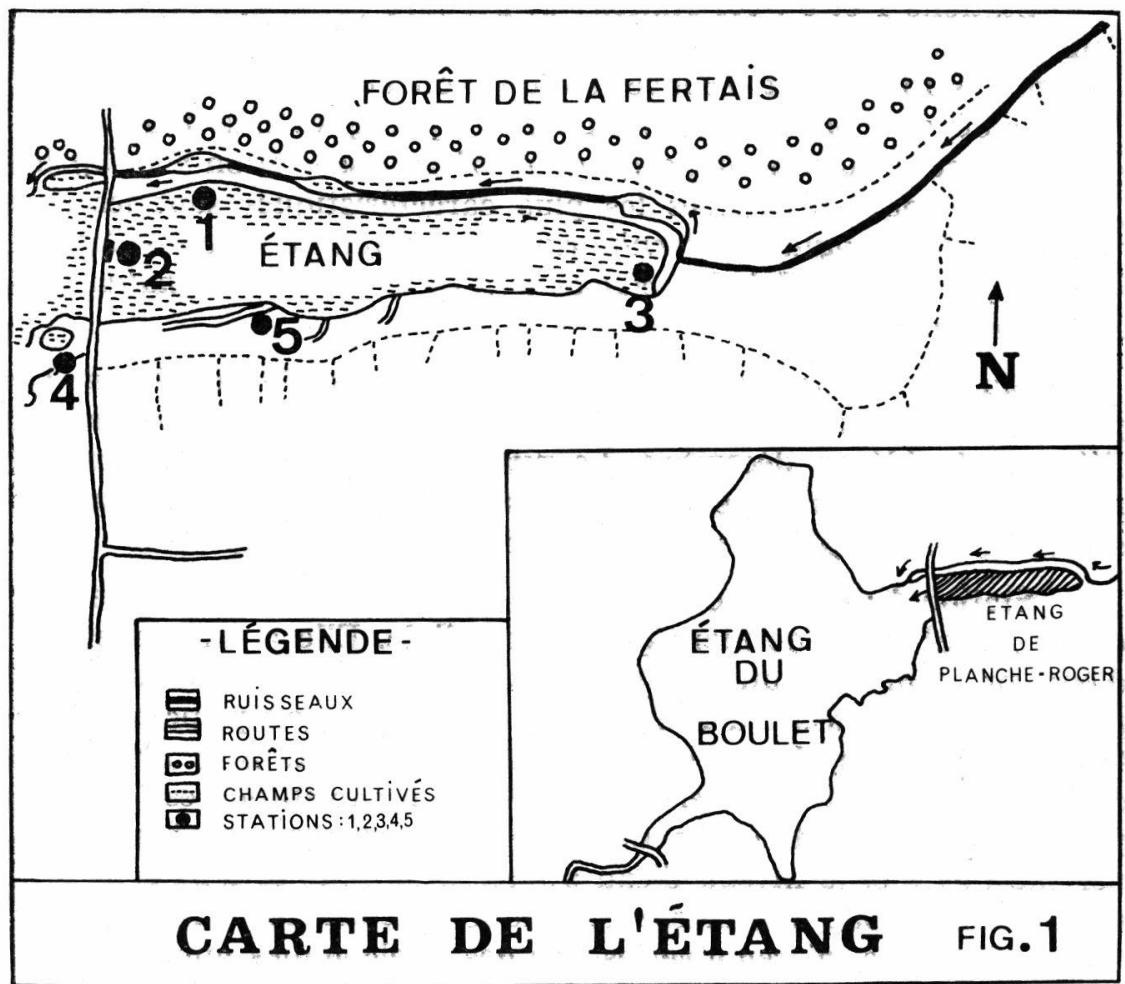

riophyllum alterniflorum. Cette espèce est accompagnée de Nymphaea alba et de Potamogeton natans. Sur les zones marginales les grands hélophytes constituent le Scirpeto-Phramitetum bien représenté à la bonde et à la queue de l'étang. Cette grande association comprend trois espèces principales : Scirpus lacustris, Equisetum limosum et Phalaris arundinacea. Son évolution mène insensiblement à un Magnocaricetum très important. Les plantes caractéristiques de cette zone sont les suivantes : Carex vesicaria et Carex paniculata. Dans la dernière zone supérieure marginale, nous notons la prédominance du Molinietum Coerulae Atlanticum avec les espèces suivantes : Molinia coerulea, Carum verticillatum, Juncus sylvaticus, Erica tetralix et Hydrocotyle vulgaris. Cette association constitue la dernière ceinture de végétation avant les formations arborescentes ou arbustives de l'Aulnaie sur la rive sud, du Quercetum sur la rive nord.

L'alimentation en eau plus ou moins régulière de nos milieux, leur profondeur, la diversité et le nombre de groupements végétaux aquatiques nous ont permis de choisir les stations suivantes : 
- Stations 1 et 3 : ces stations de bordure (nord et sud) ont une faible pente et une profondeur d'eau moyenne pouvant atteindre $50 \mathrm{~cm}$. Les fonds sont recouverts d'une couche de vase dont l'épaisseur ne dépasse pas $40 \mathrm{~cm}$. Les plantes aquatiques très nombreuses de mai à septembre font partie d'une riche association : le Myriophylletum dont les espèces les plus caractéristiques sont les suivantes : Myriophyllum alterniflorum -- Nymphaea alba - Ranunculus aquatilis - Helodea canadensis - Potamogeton natans Polygonium amphibium.

- Station 2 : cette station est située à la bonde de l'étang. La profondeur des eaux n'est jamais inférieure à $75 \mathrm{~cm}$ même lorsque l'étang est vidé en décembre. Le niveau moyen est de $1,5 \mathrm{~m}$. Le Myriophylletum est présent mais nous ne retrouvons que les espèces suivantes : Myriophyllum alterniflorum - Nymphaea alba.

- Station 4 : c'est une petite mare, ancien trou de bombe rempli d'eau datant de la dernière guerre mondiale. Elle a une superficie d'environ $50 \mathrm{~m}^{2}$ avec une profondeur variant de $50 \mathrm{~cm}$ à $1 \mathrm{~m}$. Bordée par une Aulnaie assez dense, elle est pauvre en groupements végétaux. Le Myriophylletum est faiblement représenté avec une seule espèce : Myriophyllum alterniflorum. Cette petite station est envahie par les eaux de l'étang du Boulet en février et mars. Durant la période estivale, le niveau d'eau s'abaisse de part et d'autre. Cette mare devient donc une masse aquatique indépendante; les eaux ne sont ni brassées, ni renouvelées par des quelconques apports extérieurs si l'on excepte les précipitations atmosphériques. La végétation supérieure aquatique étant peu abondante, les eaux sont peu colorées car non chargées en matières organiques de décomposition.

- Station 5 : elle est constituée par de petits marécages réunis entre eux et communiquant avec l'étang par de nombreux canaux. Cette station régulièrement inondée en hiver s'assèche progressivement au début de l'été. Bien protégée par une Aulnaie assez dense, elle est peu exposée aux vents d'ouest et du nord et se trouve ensoleillée durant la plus grande partie de la journée. On y trouve de riches groupements végétaux. La plus importante association de plantes aquatiques est un Molinietum Coeruleae Atlanticum comportant les espèces suivantes : Molinia coerulea - Juncus silvaticus - Hydrocotyle vulgaris - Erica tetralix - Potentilla tormentilla - Lotus uliginosus - Salix aurita - Salix atrocinerea Galium palustre.

\section{Conclusions.}

A la lumière de cette description, il semble déjà se dégager un caractère «fluctuant» dans nos différents types de station : c'est la permanence de l'eau. 
Dans la station 2 (bonde de l'étang), l'eau est toujours présente: même lorsque notre masse aquatique est vidée en décembre, il reste toujours une épaisseur d'eau d'environ $50 \mathrm{~cm}$. Quant aux stations de bordure 1 et 3 , elles disparaissent à la fin de l'année lors dur vidage de l'étang.

En ce qui concerne la station 5 (petits marécages) nous sommes en présence de milieux qui s'assèchent en été. Comme le souligne ViLLERET [1953], on y rencontre toujours une végétation abondante, sinon luxuriante. La température semble être le facteur décisif (milieux peu profonds et bien abrités). Etant donné la plus grande absorption des radiations calorifiques au niveau de la vase dans cette station, les populations algales forment abondamment des organes de résistance lorsque la température atteint ses valeurs optimales. Selon l'évolution, leur période de végétation est plus ou moins longue. Ces organes de résistance supportent facilement l'assèchement et fournissent un important matériel d'ensemencement au moment du retour des pluies et aussi de la remise en eau de l'étang .

\section{Les conditions climatiques.}

La zone nord de Rennes où est situé l'étang est soumise à un climat océanique. Nous avons utilisé uniquement les relevés mensuels fournis par les services de la Météorologie Nationale de Rennes et établis par la station de pisciculture du Boulet située à deux kilomètres de nos lieux de recherche (TABLEAU 1).

En été la température moyenne s'établit vers $20^{\circ}$. La température hivernale est de $4^{\circ} \mathrm{C}$.

La hauteur des précipitations atmosphériques atteint $80 \mathrm{~mm}$ par mois en hiver. Les variations des hauteurs d'eau ne sont liées qu'en partie aux chutes de pluie. En effet, à la fin de l'année, au mois de décembre, l'étang de Planche-Roger est vidé.

\section{L'ANALYSE PHYSICO-CHIMIQUE DES MILIEUX}

Les analyses physico-chimiques définissent les variations saisonnières des facteurs de l'environnement. Elles ont été entreprises régulièrement de 1963 à 1965 . En 1962 et 1966, des dosages moins fréquents nous ont permis de vérifier les données obtenues en 1963 . 1964 et 1965. Les prises d'échantillons d'eau ont été réalisées une fois par mois. Nous aurions pu effectuer des analyses plus rapprochées (tous les huit jours par exemple). En effet, le brassage des eaux, les remous de la vase peuvent provoquer d'un jour à l'autre des modifications dans le taux des substances dissoutes et en sus- 
pension. A ce moment, la représentation graphique du phénomène laisserait apparaître une courbe en dents de scie difficile à interpréter. Cependant, les recherches sur la typologie saisonnière des eaux douces ont montré que l'allure générale de la courbe-cnveloppe des variations de la concentration en sels n'était pas modifiée. On peut donc considérer qu'une analyse physico-chimique mensuelle suffit pour définir nos milieux.

\section{1. - La transparence des eaux.}

Nous avons apprécié la turbidité des eaux uniquement à la bonde de l'étang (station 2). Nous avons utilisé le disque de Secchi modifié par Nakamura [1954].

La turbidité des eaux atteint ses plus fortes valeurs de septembre à novembre (TABLEAU 1).

\section{2. -- La température.}

J es mesures de surface ont été lues sur un thermomètre ordinaire immergé durant une minute. En profondeur, nous avons utilisé le thermomètre classique à renversement.

\section{1. -- VARIATIONS ANNUELLES.}

L'étang de Planche-Roger peut être considéré, si l'on s'en rapporte aux données de Dussart [1966] comme une masse aquatique polimictique tempérée de troisième ordre. La température dépasse $+4^{\circ}$ en période estivale. En outre, cet étang peu profond ne présente pas à proprement parler de stratification stable avec établissement d'un épilimnion superficiel et d'un hypolimnion de profondeur. Cependant, le faible volume d'eau et le grand étalement de surface montrent qu'il peut être influencé par les variations de certains facteurs externes tels que l'ensoleillement et les vents. En effet, durant les journées calmes de l'été l'apport de chaleur se cantonne aux couches d'eau superficielles. Nous avons noté en juillet $1963+16^{\circ}$ au niveau de la vase et $+22^{\circ}$ dans les eaux superficielles. Il est bien évident que les eaux de surface se réchauffent beaucoup plus vite en période de forte insolation. Par contre, en période hivernale les longs moments de refroidissement permettent d'obtenir des températures plus faibles en surface. Les différences entre couches d'eau superficielle et profonde sont cependant moins élevées qu'en été; nous avions en $19632^{\circ}$ en profondeur au niveau de la vase $(80 \mathrm{~cm})$ au mois de janvier et $2^{\circ}$ en surface. Enfin, durant une grande partie du printemps, le vent et les abondantes précipitations atmosphériques participent très énergiquement au brassage des eaux. Ainsi, nous notons en mars $1963: 6^{\circ}$ dans les eaux superficielles et $7^{\circ}$ à la bonde au niveau de la vase $(1,80 \mathrm{~m}$ de hauteur $)$. 


\section{2. - VARIATIONS LOCALES.}

Dans le détail des stations que nous avons choisies, les températures relevées pour les petits marécages (station 5) sont constamment plus élevées que dans les autres biotopes à un même moment de la journée.

Ces résultat: peuvent s'expliquer par la faible profondeur des eaux, la meilleure protection de cette station contre les vents et une plus importante absorption des radiations calorifiques au niveau de la vase.

\section{3. — Le pH.}

Nous avons utilisé la méthode électrométrique.

Les mesures sont effectuées directement sur le terrain. Pour éviter tout déséquilibre ionique (départ du gaz carbonique), nous emprisonnons l'électrode dans un tube bouché contenant la solution; l'échantillon d'eau est ainsi mis à l'abri de l'air extérieur.

\section{- Résultats.}

Tous nos résultats sont consignés dans le tableau 3.

Les valeurs moyennes sont acides; elles se situent entre 6,2 et 6,8 . Durant l'été elles peuvent être supéricures à 7,0 . Cette hausse peut s'expliquer par l'augmentation de la teneur en bicarbonate et carbonate responsables de l'alcalinité totale de nos milieux.

\section{L'oxygène dissous.}

L'oxygène des eaux de nos stations traduit un équilibre avec l'atmosphère mais elle est aussi réglée par l'activité photosynthétique des végétaux aquatiques et les échanges respiratoires de tous les organismes vivants de nos biotopes.

Nous utilisons la méthode de Wrnkler d'après le:; données de Brajnikov, Francis-Bouf et Romanovsky.

Pour les prises d'échantillon nous avons adopté l'appareil VruleRET [1953] afin d'éviter le mélange des couches d'eau voisines.

\section{- Résultats.}

Tous nos résultats sont consignés sur le tableau 2. Ils sont évalués en $\mathrm{mg} / 1$ et en pourcentage de saturation. Ce \% est important car il représente la quantité d'oxygène dissous à la température d'observation.

En période printanière et hivernale, les teneurs en $0_{2}$ sont maximales. Ainsi, en février 1963, à la bonde de l'étang (station 2) la concentration en oxygène atteint $20,9 \mathrm{mg} / \mathrm{l}$, soit un taux de saturation de $160 \%$. En période estivale, les teneurs en $0_{2}$ sont minimales. Ainsi, en août 1963, nous notons $5 \mathrm{mg} / \mathrm{l}$ avec un taux de saturation de $50 \%$. 


\section{5. - Le gaz carbonique libre.}

Le gaz carbonique libre de l'eau est neutralisé par un excès de soude. On détermine cet excès à l'aide d'une solution d'acide chlorhydrique titré [RoDIER, 1966].

Nous avons utilisé pour les prises l'appareil simple décrit par Villeret [1953] dont le dispositif permet d'effectuer la manipulation à l'abri de l'air extérieur. Le $\mathrm{CO}_{2}$ est, en effet, un gaz s'échappant très facilement de l'eau dès que sa tension dépasse la tension d'équilibre avec le gaz carbonique atmosphérique.

\section{- Résultats (Tableau 2).}

Nous constatons que le gaz carbonique présente une stratification inverse de celle de l'oxygène. Les teneurs maximales s'observent, en effet, à la fin de l'été et au début de l'automne.

\section{L'alcalinité totale.}

Elle désigne la concentration de l'eau en carbonates $\left(\mathrm{CO}_{3}{ }^{-}\right)$et bicarbonates $\left(\mathrm{CO}_{3} \mathrm{H}^{-}\right)$alcalins. Dans nos milieux, les eaux acides ( $\mathrm{pH}$ souvent inférieur à 7,0 ) contiennent surtout des bicarbonates et peu de carbonates.

Le dosage est basé sur la neutralisation d'un certain volume d'eau par un acide minéral titré $\mathrm{ClH}$ ) en présence d'un indicateur coloré : la phénolphtaléine pour $\mathrm{CO}_{3}$ - et le vert de bromocrésol pour l'ion $\mathrm{CO}_{3} \mathrm{H}$-. L'hélianthine n'a pas été utilisé car il était impossible d'apprécier son virage du jaune au rose-orangé surtout lorsque les caux sont très brunes et chargées de matières organiques (composés humiques).

Pour $100 \mathrm{ml}$ d'eau, soit $X_{1}$, le nombre de $\mathrm{ml}$ de CIH N/20 versés pour l'obtention du virage de la phénolphtaléine (rose à l'incolore). $\mathrm{CO}_{3}-\mathrm{mg} / \mathrm{l}=\mathrm{X}_{1} \cdot \mathbf{1 5}$.

Soit $\mathrm{X}_{2}$ le nombre de $\mathrm{ml}$ de $\mathrm{ClH} \mathrm{N} / 20$ versés pour obtenir le virage du vert de bromocrésol (bleu au jaune) $\mathrm{CO}_{3} \mathrm{H}-=\mathrm{X}_{2} \cdot 30,5$.

- Résultats (Tableau 3).

Dans tous nos biotopes l'alcalinité à la phénolphtaléine est nulle et non mesurable. Il y a donc peu de carbonates alcalins. Les eaux de nos stations ne sont pratiquement tamponnées que par des bicarbonates (alcalinité positive au vert de bromocrésol).

L'alcalinité totale est faib'e en hiver (janvier et février); elle atteint son maximum en été (mai et juin). Cette hausse estivale peut s'expliquer. En effet, l'intense activité photosynthétique des végétaux aquatiques peut se faire aux dépens du $\mathrm{CO}_{2}$ des bicarbonates. Dans ces conditions, des ions $\mathrm{CO}_{3}^{-}$apparaissent; ces ions qui, en solution d'égale concentration, sont plus alcalins que les bicarbon:'es provoquent une montée du pH et de l'alcalinité totale. 


\section{7. - Les Anions et les Cations.}

Les analyses ont été réalisées sur des échantillons d'eau de surface.

\section{1. - Les Sulfates.}

En milieu alcalin $(\mathrm{ClH})$, l'anion $\mathrm{SO}_{4}=$ est précipité sous forme de sulfate de benzidine insoluble. Nous lavons le précipité et l'hydrolysons à chaud. L'acide sulfurique libéré est dosé par une base titrée $(\mathrm{NaOH})$ en présence d'un indicateur coloré (phénolphtaléine).

Nous utilisons la technique de Gedrorts [1963] mais nous la modifions légèrement. Les dosages sont réalisés de la manière suivante : nous évaporons au $1 / 4$ un litre d'eau. Nous ajoutons $1 \mathrm{ml}$ de CIH concentré et maintenons à l'ébullition durant 10 minutes. Nous versons alors $10 \mathrm{ml}$ de benzidine à $10 \%$ et $5 \mathrm{ml}$ de chlorhydrate d'hydroxylamine à $1 \%$ réactif qui permet de supprimer l'interférence des ions ferriques. Tout le fer passe à l'état de sel ferreux soluble. Nous agitons et laissons reposer 20 minutes hors de la flamme. Le précipité de sulfate de benzidine se forme. Il est rassemblé sur un filtre sans cendres adapté à un Buchner. Nous faisons le vide de façon à laver le précipité. Nous plaçons le filtre dans un vase à saturer et $\mathrm{y}$ ajoutons $50 \mathrm{ml}$ d'eau distillée. Nous faisons bouillir cinq minutes. L'acide sulfurique libéré par hydrolyse est titré par une solution de soude $\mathrm{N} / 20$ en présence de phénolphtaléine à $1 \%$.

- $1 \mathrm{ml}$ de $\mathrm{NaOH} \mathrm{N} / 20$ correspond à $2,4 \mathrm{mg}$ de $\mathrm{SO}_{4}=$.

L'adjonction d'hydroxylamine élimine les sels ferriques qui gênent la totale précipitation des sulfates. Tout le fer présent dans l'échantillon passe à l'état de fer ferreux soluble. En outre, l'ion $\mathrm{SO}_{4}=$ est le seul à pouvoir s'unir à la benzidine et donner un précipité insoluble. Or, avec la méthode gravimétrique classique, il peut y avoir coprécipitation de la silice, le silicate de baryum se formant très rapidement.

\section{- Résultats (Tableau 4).}

Les variations annuclles enregistrées présentent une évolution saisonnière identique à celle de l'oxygène. Les taux sont maxima au début de l'hiver; les valeurs minimales se situant généralement en plein été (juin et juillet).

\section{2. - Les Nitrates.}

Les nitrates proviennent en partie de la décomposition aérobie des matières organiques azotées. Il ne faut cependant pas négliger l'apport des ions $\mathrm{NO}_{3}^{-}$venant du lessivage des champs riverains de l'étang lorsque les précipitations atmosphériques sont abondantes. 
A l'aide d'un catalyseur approprié, nous réduisons les nitrates en nitrites. Ces derniers sont ensuite dosés par la méthode colorimétrique au N(1-Naphtyl).

Nous utilisons une technique modifiée d'après les travaux de Wooley, Hicks et Haguenau [1960] sur les extraits végétaux. Le mode opératoire peut se résumer de la façon suivante : à $10 \mathrm{ml}$ d'eau nous ajoutons $100 \mathrm{mg}$ d'un réactif dont la composition est définie comme suit :

— Acide sulfanilique $\ldots \ldots \ldots \ldots \ldots, 0,8 \mathrm{~g}$

- Acide citrique ............. 7,5 g

- Sulfate de Mangan`se .......... 2,0 g

- Poudre de Zinc ............ 2,0 g

- N(1-Naphtyl) $\ldots \ldots \ldots \ldots \ldots \ldots \ldots, 0,4 \mathrm{~g}$

Après avoir acidifié le milieu par $0,5 \mathrm{ml}$ d'acide acétique concentré, nous laissons en contact trois minutes en agitant régulièrement. Nous centrifugeons. Les nitrates sont alors réduits en nitrites. L'intensité de la coloration rose qui se développe est proportionnelle a la quantité de nitrites et susceptible d'un dosage photocolorimétrique. Ce procédé permet en réalité de doser la somme $\mathrm{NO}_{3}+\mathrm{NO}_{2}$. Or, dans nos stations, les teneurs en nitrites sont faibles et non dosables par le N(1-naphtyl). Nous pouvons donc admettre que nos résultats n'indiquent pratiquement que des concentrations en nitrates.

Par ce procédé, nous pouvons effectuer en peu de temps plusieurs dosages sur de nombreuses prises d'essai et n'utiliser à chaque fois qu'un faihle volume d'eau. Enfin, le réactif est très sensible et permet de doser de petites quantités de nitrates (à partir de $0,05 \mathrm{mg} / \mathrm{l}$ en $\mathrm{NO}_{3}{ }^{-}$).

\section{-.- Résultats (TABleau 5).}

Les valeurs exprimées en $\mathrm{NO}_{3}$ - oscillent entre 2,1 et $3,0 \mathrm{mg}$ par litre de milieu. Les taux de nitrates sont maxima en hiver et au début du printemps (février et mars). Nous observons, par contre, une concentration minimale en automne.

\section{3. - Les Chlorures.}

Nous utilisons la méthode classique de Mohr [Rodier, 1966]. Ainsi, le taux de chlorures que nous exprimons en ClNa est donné par la formule suivante : $\mathrm{CINa} \mathrm{mg} / \mathrm{l}=\mathbf{5 8 , 5} . \mathrm{v}$; v représente le nombre de $\mathrm{ml}$ de $\mathrm{NO}_{3} \mathrm{Ag} \mathrm{N} / 10$ versés au cours du dosage volumétrique.

\section{- Résultats (TABLEaU 5).}

Pratiquement le minimum de chlorures se situe à $30 \mathrm{mg} / \mathrm{l}$ mais le taux maximum peut atteindre $60 \mathrm{mg} / \mathrm{l}$. Il semble toutefois qu'il n'y uit pas de cycle saisonnier bien défini comme pour les sulfates 
et les nitrates. Les variations semblent être occasionnées soit par les fortes précipitations atmosphériques de l'hiver, soit par les évaporations intenses des solutions durant la période estivale.

\section{4. - L'Azote Ammoniacal.}

Nos dosages ont porté sur deux formes d'azote ammoniacal :

L'azote ammoniacal libre : il provient principalement de la réduction des nitrates et des nitrites.

L'azote ammoniacal albuminoïde : cette fraction semble être issue des groupements $\mathrm{NH}_{2}$ constitués par les peptides et polypeptides des matières organiques.

Les deux fractions (libre et albuminoïde) sont distillées en milieu alcalin puis dosées volumétriquement en présence d'un indicateur coloré (Tashiro : bleu de méthylène et rouge de méthy!e).

Nous avons apporté quelques modifications à la méthode classique.

7.4.1. -- Dosage de la fraction libre. - $500 \mathrm{ml}$ d'eau sont placés dans un ballon de distillation. Nous ajoutons $10 \mathrm{ml}$ de so'ution tampon $(\mathrm{pH}=7,4)$. Nous recueillons $100 \mathrm{ml}$ de distillat que l'on reçoit dans $50 \mathrm{ml}$ d'acide borique à $2 \%$; nous complétons à $200 \mathrm{ml}$ avec de l'eau distillée exempte d'ammoniaque. Nous dosons ensuite par volumétrie avec de l'acide sulfurique $\mathrm{N} / 50$.

7.4.2. - Dosage de la fraction albuminoüde. - On conserve la liqueur restante après dosage de la fraction libre. On ajoute $50 \mathrm{ml}$ d'une solution alcaline de $\mathrm{MnO}_{4} \mathrm{~K}$ (préparée suivant la technique classique.

\subsection{3. - Résultats (TABlEAu 6).}

Evolution de la fraction $\mathrm{N}_{\mathrm{NH}} \mathrm{NH}_{3}$ libre. - Les teneurs oscillent entre 0,02 et $1,0 \mathrm{mg} / 1$ en $\mathrm{N}^{-\mathrm{NH}_{3}}$. Les valeurs maximales sont atteintes en septembre.

Evolution de la fraction albuminoïde. -- Les valeurs minimales atteignent $0,1 \mathrm{mg} / \mathrm{l}$ en $\mathbf{N} . \mathrm{NH}_{3}$. Les taux maxima se situent autour de $0,8 \mathrm{mg} / \mathrm{l}$. Cependant, nous ferons une exception pour les eaux de la mare isolée (station 4), la plus forte tencur atteignant $1,9 \mathrm{mg} / 1$. Nous sommes alors en droit de nous demander si les troupeaux venant très régulièrement s'abreuver ne sont pas rendus responsables de cette hausse particulière.

\section{7.ð. - - LES PHOSPHATES.}

Nous n'avons dosé que les phosphates solubles.

Les phosphates en solution nitrique et en présence de métavanadate et de molybdate d'ammonium donnent un complexe jaune de phosphomolybdovanadate d'ammonium susceptible d'un dosage colorimétrique. 
Nous avons modifié la méthode classique décrite par PinTa [1962] en nous inspirant des données de Proft [1964] et de Quinhan [1955]. Le mode opératoire peut se résumer de la façon suivante : dans une ampoule à décanter nous disposons $200 \mathrm{ml}$ d'eau à analyser, nous ajoutons $5 \mathrm{ml}$ de molybdate d'ammonium à $10 \%$ et $5 \mathrm{ml}$ de métavanadate d'ammonium à $1 \%$. Nous acidifions par $5 \mathrm{ml}$ d'acide nitrique $4 \mathrm{~N}$. Nous laissons reposer cinq minutes. Nous versons dans l'ampoule $10 \mathrm{ml}$ d'alcool amylique. Après avoir agité l'ampoule sans créer d'émulsion, nous laissons reposer de nouveau durant cinq minutes. Le complexe formé très stable et de couleur jaune passe dans l'alcool. Nous éclaircissons la phase supérieure par $0,5 \mathrm{ml}$ d'alcool éthylique absolu. La silice présente parfois en grandes quantités (10 à $15 \mathrm{mg} / \mathrm{l})$ ne gêne pas le dosage car le silicomolybdate formé reste dans la phase aqueuse.

\section{- Résultats (Tableau 4).}

Les quantités sont faibles pour l'ensemble des stations. Nos milieux ne sont donc pas tamponnés par des phosphates. Les valeurs maximales s'observent de la fin de l'automne au début du printemps; elles atteignent alors $0,12 \mathrm{mg} / \mathrm{l}$. Les concentrations les plus faibles se situent en juin et juillet; elles sont inférieures à $0,05 \mathrm{mg} / \mathrm{l}$ et sont done difficiles à doser avec notre méthode.

\section{6. - Le Calcium.}

Nous awons utilisé la technique classique gravimétrique.

\section{- Résultats (Tableau 7).}

Les teneurs évaluées en $\mathrm{CaO}$ oscillent entre $10 \mathrm{mg} / 1$ et $24,3 \mathrm{mg} / \mathrm{I}$. En outre, il ne semble pas y avoir de cycle annuel bien défini comme nous avons pu le voir à propos des sulfates et des nitrates par exemple. Nous sommes bien en présence d'eaux acidophi'es, peu minéralisées.

\section{- Remarques.}

L'étang de Planche-Roger, réserve de pêche est chaulé tous les deux ans au mois de juin. Cette chaux est un élément plastique qui participe au maintien du $\mathrm{pH}$ des eaux nécessaire d'ailleurs au bon développement des alevins. En effet, en période printanière notre étang est encore soumis à des décompositions organiques par suite de l'élévation brutale de la température. Il se produit une augmentation du taux de gaz carbonique. Le calcium qui est en large excès se combine facilement à $\mathrm{CO}_{2}$ pour former des carbonates plus alcalins que les bicarbonates. Les ions $\mathrm{Ca}++$ en excès permettent donc de remonter le $\mathrm{pH}$ devenu assez bas par suite d'une forte quantité de gaz carbonique dissous. 
Au moment du chaulage, il se produit bien une augmentation du taux de calcium le long de la rive sud $(110 \mathrm{mg} / \mathrm{l}$ en $\mathrm{CaO}$ au lieu de $20 \mathrm{mg} / \mathrm{l})$. Cette hausse est éphémère. Huit jours plus tard, nous notons au même endroit $24 \mathrm{mg} / 1$ en $\mathrm{CaO}$ et il n'y avait eu à ce moment aucun écoulement d'eau important à la bonde de l'étang. Ce phénomène a déjà été observé par Villeret [1953] dans les mares de Bretagne. Il semble que la disparition assez rapide de la chaux ne soit pas liée à son utilisation immédiate par les organismes vivants mais laisse entrevoir des phénomènes d'adsorption qui aboutissent finalement à un équilibre stable.

\section{7. - Le Magnésium.}

Comme le calcium, le magnésium est un élément de la dureté de l'eau. Il est, d'autre part, lié à des substances organiques telles que la chlorophylle des végétaux.

Nous avons utilisé la méthode classique par complexométric.

-. Résultats (Tableau 7).

Au cours de l'année, les variations du taux de magnésium suivent assez généralement celles du calcium mais avec des contrastes beaucoup moins marqués. Il ne semble pas y avoir de cycle annuel bien défini.

Les quantités observées, évaluées en MgO sont faibles. Les concentrations oscillent, en effet, entre $5,5 \mathrm{mg} / \mathrm{l}$ et $12 \mathrm{mg} / \mathrm{l}$. Très supéricures à celles des eaux de tourbière [VILLERET, 1953] elles montrent que nos milieux sont peu minéralisés et se rapprochent des eaux du type oligotrophe. Les variations annuelles semblent maximales en période estivale (juillet et août). Les décompositions déjà très importantes à la fin de l'été pourraient peut-être expliquer l'enrichissemeent du milieu en ions $\mathbf{M g}^{++}$.

\section{8. - La Silice.}

Cette silice existe dans les eaux à l'état dissous (ionique) mais aussi sous forme colloïdale. Nous avons utilisé la méthode classique gravimétrique.

\section{- Résultats (Tableau 8).}

Les valeurs oscillent entre $2,0 \mathrm{mg} / \mathrm{l}$ et $20,0 \mathrm{mg} / \mathrm{l}$ en $\mathrm{SiO}_{2}$. Les taux minima sont atteints en été. Les concentrations maximales sont obtenues en hiver (décembre et janvier). 
7.9. - Le Fer total.

Ce métal prend beaucoup d'importance chez les végétaux aquatiques. La chlorophylle en exige pour la synthèse de ses noyaux tétrapyrroliques et certaines algues (Cyanophycées et Desmidiées) peuvent fixer du fer dans leurs membranes cellulaires. Nous utilisons la méthode classique gravimétrique.

\section{- Résultats (Tableau 8).}

Les teneurs maximales sont notées à la fin de l'été. Elles atteignent alors 18,0 à $20 \mathrm{mg} / \mathrm{l}$ en $\mathrm{Fe}_{2} \mathrm{O}_{3}$. Les valeurs minimales s'observent en hiver et au début du printemps; elles sont de l'ordre de 2,0 à $5,0 \mathrm{mg} / 1$.

\subsection{0. — LES OLIGO-ÉLÉMENTS.}

Pour de nombreux auteurs, les oligo-éléments sont indispensables a faible dose pour la croissance des populations algales. RodHE [1949] montre que la carence du milieu en molybdène provoque un net ralentissement des cultures de Scenedesmus; il ajoute que quelques traces de Mo apportées aux algues $(2,0$ à $5 \mu \mathrm{g} / 1)$ suffisent à améliorer le rendement cellulaire. Goldman [1960] dans ses travaux sur la productivité primaire du phytoplancton des lacs de Californie, montre que l'adjonction au milieu naturel de $1 \mathrm{mg} / \mathrm{l}$ de Mo permet de doubler le quotient photosynthétique des algues présentes. Il est vrai que l'importance de ce micro-élément dans l'activité du métabolisme azoté est bien établie (cofacteur essentiel de la nitrate-réductase permettant la transformation des nitrates en nitrites). De récents travaux entrepris par Williams et MounT [1965] révèlent l'importance d'autres oligo-éléments comme le cobalt et le zinc. Ces auteurs admettent, en effet, que des apports en Co et $\mathrm{Zn}$ peuvent augmenter le rendement cellulaire de quelques algues d'eau douce (Oocystis sp., Euglena acus, Trachelomonas volvocina et la majorité des Oscillatoria). Cependant, l'ensemble des recherches sur la dynamique des populations algales indiquent que de trop fortes concentrations entre oligo-éléments sont néfastes. Mount [1965] le signale; Mac Combie [1953] précise, d'autre part, que le cuivre se révèle un algicide puissant lorsque son taux est trop élevé dans les eaux.

Aussi, nous a-t-il paru intéressant de connaître les variations de concentration en oligo-éléments dans nos stations. Nous nous sommes limités au dosage du molybdène, du cuivre et du cobalt.

\subsection{1.-- Techniques d'extraction et de dosage.}

Le Molybdène. - Nous avons adapté au miliè aquatique la méthode proposée par Pinta et Sauerbeck [1962] pour les extraits végétaux. 
- Le Cobalt. - L'extraction du cobalt est identique à celle du molybdène (évaporation de deux litres d'eau puis destruction de la matière organique par l'eau oxygénée). On effectue cependant une extraction préalable des sels de fer par $40 \mathrm{ml}$ d'éther éthylique en utilisant la technique de Burries et Galdego [1953].

- Le Cuivre. - Nous avons adopté aux eaux douces la technique proposée par CAPELLE $[\mathbf{1 9 6 0}]$ pour les sols et les extraits végétaux. 7.10.2. - Résultats (TABLEA 3). - Les dosages ont été effectués uniquement sur les eaux superficielles de la station 2 (bonde de l'étang).

En ce qui concerne le molybdène et le cobalt, les valeurs sont souvent très faibles et inférieures à $5,0 \mathrm{~m} \mu \mathrm{g} / \mathrm{l}$. Les teneurs maximales sont notées en période automnale.

Pour le cuivre, les taux sont également bas et ne dépassent pas $10 \mathrm{~m} \mu \mathrm{g} / \mathrm{l}$ (période post-estivale).

\section{III. - RAPPEL DE LA TYPOLOGIE \\ DES EAUX DOUCES BRETONNES \\ ET TYPOLOGIE DES EAUX DE L'ETANG ÉTUDIÉ}

L'analyse physico-chimique des eaux hretonnes a permis dans de précédentes recherches [SAvouné, 1964, 1966, 1967, 1968, 1969] de distinguer deux types d'étang :

1. - Type à tendance Eutrophe. - Les eaux de ce type se caractérisent dans leur ensemble par une prédominance des ions $\mathrm{Ca}^{++}$ (30 à $60 \mathrm{mg} / \mathrm{l})$. Les autres éléments sont bien représentés : $\mathbf{M g}^{++}$ $(4$ à $10 \mathrm{mg} / \mathrm{l}) ; \mathrm{Na}^{+}(5$ à $15 \mathrm{mg} / \mathrm{l}) ; \mathrm{K}+(2$ à $9 \mathrm{mg} / \mathrm{l}) ; \mathrm{SO}_{\mathbf{4}}-(10$ à $30 \mathrm{mg} / \mathrm{l})$. Le $\mathrm{pH}$ est voisin de la neutralité.

2. - Type Oligotrophe. - Ce type se trouve généralement dans les étangs de forêt de Bretagne : étangs de la forêt de Paimpont en Ille-et-Vilaine, étangs de la forêt de Quénécan dans le Finistère. Les eaux sont plus isovalentes que celles du type précédent. Le cation $\mathrm{Ca}^{+}+$est faiblement représenté $(2,5$ à $10 \mathrm{mg} / \mathrm{l})$. Il en est ainsi pour les autres éléments dissous : $\mathrm{Mg}++(2$ à $9 \mathrm{mg} / \mathrm{l}) ; \mathrm{Na}^{+}(2$ à $5 \mathrm{mg} / \mathrm{l})$; $\mathrm{K}+(0,7$ à $2 \mathrm{mg} / \mathrm{l}) ; \mathrm{SO}_{4}^{--}(1,5$ à $10 \mathrm{mg} / \mathrm{l})$. Le $\mathrm{pH}$ souvent compris entre 5,5 et 7,0 peut cependant, sous l'effet de l'assimilation chlorophyllienne des végétaux aquatiques dépasser la neutralité (valeurs supérieures à 7,5 ).

3. - Typologie des caux de l'étang étudié. - A la lumière des résultats de l'analyse physico-chimique des eaux, nous pouvons considérer que l'étang de Planche-Roger est du type «Oligotrophe $\gg$. La masse aquatique est faiblement minéralisée et essenticllement tamponnée par le $\mathrm{CO}_{2}$ libre et des bicarbonates, non par des phosphates comme dans le cas des eaux Eutrophes. 


\section{IV. - LES VARIATIONS DES FACTEURS PHYSICO-CHIMIQUES DANS LES STATIONS;} TRAITEMENT MATHEMATIQUE DES DONNEES

Dans les paragraphes précédents, nous avions précisé, pour chaque type de station choisie $(1,2,3,4,5)$ les fluctuations saisonnières des facteurs environnants. Nous les connaissons donc bien mais ontelles une signification?

D'autre part, existe-t-il une similitude de variation entre plusieurs facteurs physico-chimiques? Enfin, peut-on trouver des différences d'un biotope à l'autre dans les fluctuations saisonnières des facteurs environnants? Si oui, sommes-nous capables de les définir et de les expliquer?

Il était donc intéressant d'exprimer les «correspondances»entre facteurs physico-chimiques et biotopes. Nous avons choisi cette méthode pour les raisons suivantes :

- Elle ne nécessite pas de gros échantillonnages.

- Elle peut traiter un grand nombre de données.

- Elle permet la représentation simultanée et duale des stations et des facteurs environnants.

— Elle fait perdre très peu d'information en cours de calcul.

\section{La technique expérimentale.}

Elle consiste donc à représenter simultanément l'ensemble des stations et des facteurs physico-chimiques environnants dans un espace à faible dimension tout en respectant au mieux les données. initiales.

Considérons troits stations : $\mathrm{St}_{1}, \mathrm{St}_{2}, \mathrm{St}_{3}$ et $n$ facteurs $\mathrm{F}_{1}, \mathrm{~F}_{2}, \ldots$ $F_{n}$. Chaque station peut être représentée par une combinaison linéaire de ces $n$ facteurs :

- Pour la station 1: $\mathrm{F}_{1}$ aura la valeur $a_{1} ; \mathrm{F}_{2}$ aura la valeur $a_{2}$; $F_{n}$ aura la valeur $a_{n}$.

- Pour la station 2 on aura : $a_{1}^{\prime}, a_{2}^{\prime} \ldots a_{n}^{\prime}$.

- Pour la station 3 on aura : $a^{\prime \prime}{ }_{1}, a^{\prime \prime}{ }_{2} \ldots a_{n}^{\prime \prime}$.

1.1. - Définition de la combinaison linéaire : on considère ainsi la suite $\left(a_{1}, a_{2}, \ldots a_{n}\right)$ pour la station 1 . Nous dirons que $R_{1}$ est une combinaison linéaire de cette suite de nombres de coefficients $Y_{\text {y }}$, $\mathrm{Y}_{2}, \ldots \mathrm{Y}_{n}$ si :

$$
\mathrm{R}_{1}=\mathrm{Y}_{1} a_{1}+\mathrm{Y}_{2} a_{2}+\ldots+\mathrm{Y}_{n} a_{n} .
$$

Or, l'ensemble des données se présente sous la forme d'un tableau (fig. 2) où chaque ligne correspond à une station et chaque colonne 
à un caractère physico-chimique donné. La combinaison la plus simple pourrait être :

$$
R_{1}=Y_{1} a_{1}+Y_{2} a_{2}+\ldots+Y_{n} a_{n} \text { avec } Y_{1}+Y_{2}=Y_{n}=1 / n \text {. }
$$

Nous aurions alors une combinaison linéaire qui représenterait la moyenne des caractères pour chaque station.

1.2. - Mise en place des stations : pour caractériser nos stations nous allons chercher les combinaisons linéaires $R_{1}, R_{2}, \ldots R_{3}$ qui donneront aux suites $\left(\mathrm{Y}_{1} a_{1}+\mathrm{Y}_{2} a_{2}+\ldots \mathrm{Y}_{n} a_{n}\right),\left(\mathrm{Y}_{1}^{\prime} a_{1}^{\prime}{ }_{1}+\mathrm{Y}_{2}^{\prime} a^{\prime}{ }_{2}\right.$ $\left.+\ldots \mathrm{Y}_{n}^{\prime} a_{n}^{\prime}\right),\left(\mathrm{Y}_{1}^{\prime \prime} a^{\prime \prime}{ }_{1}+\mathrm{Y}_{2}^{\prime \prime} a_{2}^{\prime \prime}+\ldots \mathrm{Y}_{n}{ }_{n} a_{n}^{\prime \prime}\right)$ les plus grandes variances.

Soient $\mathrm{Cl}_{1}, \mathrm{Cl}_{2}, \mathrm{Cl}_{3} \ldots \mathrm{Cl}_{q}$ les $q$ combinaisons linéaires avec $q<n$ auxquelles sont associées les suites $\mathbf{R}$

\begin{tabular}{|c|c|c|c|}
\hline $\begin{array}{l}\mathrm{Cl}_{1} \\
\mathrm{R}_{1}\end{array}$ & $\begin{array}{l}\mathrm{Cl}_{1} \\
\mathrm{R}_{2}\end{array}$ & $\begin{array}{r}\mathrm{Cl}_{1} \\
\mathbf{R}_{3}\end{array}$ & variance optimale $\sigma^{2}$ \\
\hline $\begin{array}{l}\mathrm{Cl}_{2} \\
\mathbf{R}^{\prime}{ }_{1}\end{array}$ & $\begin{array}{c}\mathbf{C l}_{2} \\
\mathbf{R}_{2}^{\prime}\end{array}$ & $\begin{array}{c}\mathrm{Cl}_{2} \\
\mathbf{R}^{\prime}{ }_{3}\end{array}$ & variance $\sigma^{\prime 2}<\sigma^{2}$ \\
\hline $\begin{array}{l}\mathrm{Cl}_{q} \\
\mathrm{R}^{\prime \prime}{ }_{1}\end{array}$ & $\begin{array}{l}\mathrm{Cl}_{q} \\
\mathrm{R}^{*{ }_{2}}\end{array}$ & $\begin{array}{c}\mathrm{Cl}_{\boldsymbol{q}} \\
\mathrm{R}^{\prime \prime}{ }_{3}\end{array}$ & variance $\sigma_{q}^{2}<\sigma^{\prime 2}$ \\
\hline
\end{tabular}

Soit $\sigma^{2}$ T la variance totale du tableau des données, nous dirons que l'on a une représentation fidèle dans un espace à $q$ dimensions si la différence $\sigma^{2} \mathrm{~T}-\left(\sigma^{2}+\sigma_{q}{ }^{2}\right)$ est inférieure aux erreurs de calcul.

Si $q=2$, ce qui est le cas le plus fréquent, on aura une représentation plane de l'ensemble des stations.

Nous construirons alors sur un axe orienté $M$ les valeurs $R_{1}, R_{2}$, $R_{3}$ correspondant à nos trois stations face aux $n$ caractères. Ainsí, pour la variance optimale, les points $R_{1}, R_{2}, R_{3}$ représentent nos biotopes $\mathbf{S t}_{1}, \mathbf{S t}_{2}, \mathbf{S t}_{3}$. Il en sera de même pour les variances suivantes : $\sigma^{\prime 2}, \sigma^{\prime \prime 2} \ldots$ les valeurs $R^{\prime}, R^{\prime \prime} \ldots$ représenteront nos diffétes stations.

1.3. - Mise en place des caractères par rapport aux stations.

Pour placer le caractère $F_{1}$ on attribuera : (fig. 2 ).

- à la station 1 : un poids égal à $p_{1}(1)$

- à la station 2 : un poids égal à $p_{1}(2)$

- à la station 3 : un poids égal à $p_{1}$ (3)

Nous plaçons alors sur un axe perpendiculaire à $\mathbf{M}$ par construction les valeurs $p_{1}(1), p_{1}(2), p_{1}(3)$. Nous obtenons à ce moment trois nouveaux points qui représentent les valeurs de $F_{1}$ pour chaque station. Nous calculons alors le centre de gravité de ces trois masses. Soit $G$ ce point. De $G$ nous abaissons par construction une perpendiculaire sur l'axe $M$. Soit $G^{\prime}$ la projection de $G_{\text {. }} G^{\prime}$ représente 

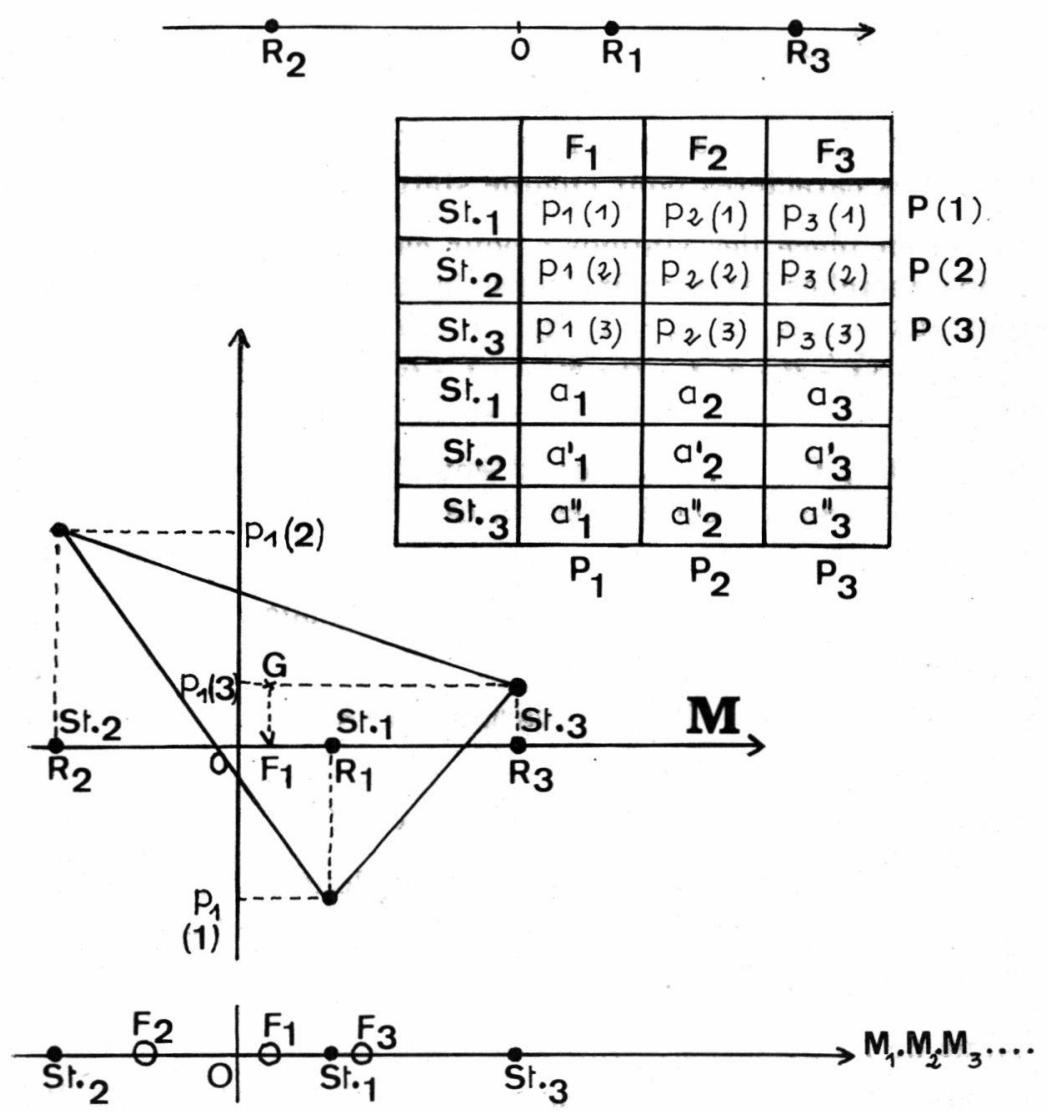

\section{ANALYSE DES CORRESPONDANCES FIG. 2}

la position de $\mathrm{F}_{1}$ par rapport à $\mathrm{R}_{1}, \mathrm{R}_{2}, \mathrm{R}_{3}$, c'est-à-dire à $\mathrm{St}_{1}, \mathrm{St}_{2}$, $\mathrm{St}_{3}$ (fig. 2).

Nous opérons de la même façon pour avoir $\mathrm{F}_{2}, \mathrm{~F}_{3}, \ldots \mathrm{F}_{n}$. Nous obtenons ainsi sur l'axe $\mathbf{M}$ une représentation plane simultanée des deux ensembles (stations et facteurs physico-chimiques) qui est une image géométrique assez fidèle des données initiales.

Le premier axe $\mathbf{M}$ correspondra aux combinaisons linéaires à variance maximale (optima!e) : $\sigma^{2}$. L'axe $\mathrm{M}_{2}$ correspondra à des combinaisons linéaires à variance forte mais inférieure à $\sigma^{2}$.

Si nous avions voulu d'abord placer les caractères par rapport aux stations puis, ensuite, les stations par rapport aux caractères, 
l'image obtenue aurait été identique en plaçant les biotopes puis ensuite les caractères par rapport aux biotopes.

\section{L Lanalyse proprement dite.}

Le traitement des données sur ordinateur concerne quatre stations aquatiques et dix caractères physico-chimiques environnants dont les variations ont été mesurées chaque mois durant deux années consécutives. Parmi les axes qui sont classés par l'ordinateur, seuls les trois premiers (variances maximales $\sigma^{2}<\sigma^{\prime 2}<\sigma^{\prime \prime 2}$ ) contiennent suffisamment d'informations pour être exploités.

Nous pouvons d'ailleurs, grâce aux deux premiers $\left(\mathbf{M}_{1}, \mathbf{M}_{2}\right)$, exprimer les nouvelles données dans un plan (deux axes perpendiculaires par construction). Nous voyons alors, sur ce graphique à deux dimensions, les images des facteurs environnants et des biotopes.

Dans une première analyse, nous avons défini les stations par rapport aux caractères physico-chimiques.

Dans une seconde analyse partielle nous avons placé les caractères par rapport aux biotopes.

Enfin, dans un troisième temps, nous avons effectué une analyse globale des données par traitements simultanés des résultats d'expérience. Nous signalerons que notre traitement des « Correspondances » n'a pu tenir compte des résultats de la station 5 (petits marécages). Les mesures mensuelles n'ont pas été faites en période estivale par suite d'une évaporation quasi-totale des eaux.

3. - Les résultats (fig. 3, 4 et 5 ).

\section{1. - L'analyse globale.}

Aspect général (fig. 3). - Nous observons tout d'abord une concentration massive de points à l'origine des deux premiers axes $\mathbf{M}_{1}$ et $\mathrm{M}_{2}$. Ce triangle de points rassemble les résultats du $\mathrm{pH}$, du calcium, du magnésium, des chlorures et des nitrates pour tous nos biotopes. Cet étirement minimum traduit peut-être un état d'équilibre permanent des eaux pour ces éléments. En effet, si nous comparons avec nos graphiques initiaux, nous constatons que les teneurs en $\mathrm{Ca}, \mathrm{Mg}, \mathrm{Cl}$ et $\mathrm{NO}_{-}$ne subissent pas de grandes variations au cours d'un cycle annuel de végétation.

Il en est tout autrement pour l'oxygène, le gaz carbonique, le fer, la silice et les sulfates. Nous voyons que $\mathrm{O}_{2}$ et $\mathrm{CO}_{2}$ sont en opposition très nette; ils évoluent en sens inverse. Il en est ainsi pour $\mathrm{O}_{2}$ et $\mathrm{Fe}$. Il y a, d'autre part, évolution parallèle entre $\mathrm{CO}_{2}$ et $\mathrm{Fe}$. Toutes ces observations concordent d'ailleurs parfaitement avec l'allure générale des courbes représentatives des variations 


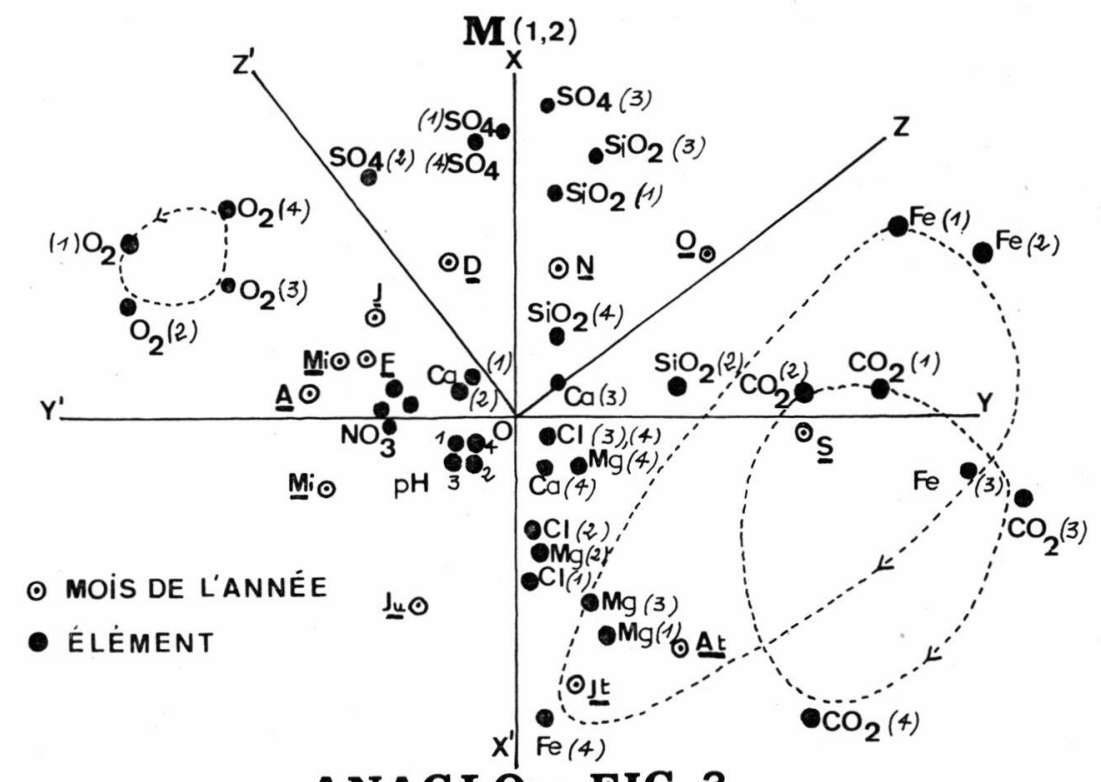

ANAGLO - FIG. 3

saisonnières de ces éléments. En effet, les valeurs maximales de $\mathrm{CO}_{2}$ et de $\mathrm{Fe}$ coïncident (période juillet-août-septembre). Les minima d'oxygène correspondent à des maxima de gaz carbonique. Ces variations peuvent s'expliquer de la manière suivante : en période estivale et automnale, la minéralisation des matières organiques provenant de la décomposition des organismes aquatiques nécessite beaucoup d'oxygène avec rejet important de $\mathrm{CO}_{2}$. Le milieu ambiant s'appauvrit en $\mathrm{O}_{2}$, devient riche en gaz carbonique et en fer soluble.

D'autre part, considérant l'ensemble de l'analyse globale, nous pouvons dire que tous nos biotopes paraissent soumis à quatre périodes successives formant un cycle bien défini :

1) Une période z'oy' (janvier à avril) : elle est caractérisée par une oxygénation intense de l'eau. Elle correspond sans doute à l'Overturn, période de mélange des eaux superficielles et des eaux profondes.

2) Une période y'ox' (mai et juin) : il n'y a aucune activité dominante. Cette période correspond grossièrement à l'été (eaux calmes, faibles précipitations).

3) Une période x'oz : elle correspond à l'établissement des décompositions organiques. Elle se caractérise par un rejet important de 
$\mathrm{CO}_{2}$ et une remise en solution des sels de fer. Elle se situe de juillet à septembre.

4) Une période zoz' : cette période (octobre à décembre), est caractérisée par une arrivée massive de silice $\left(\mathrm{SiO}_{2}\right)$ et de sulfates $\left(\mathrm{SO}_{4}^{--}\right)$. Les fortes précipitations atmosphériques du moment permettent ainsi un lessivage important par les ruisseaux forestiers d'alimentation. En outre, il est admis depuis longtemps que les eaux de pluie sont toujours très chargées en sulfates.

\section{2. - DÉComposition DE L’ANALYSE GLOBALE.}

Nous avons reproduit séparément les résultats de l'analyse globale pour chaque station. Il est, en effet, intéressant de comparer la position et la longueur des vecteurs représentant chaque facteur physico-chimique étudié (fig. 4).

Nous pouvons dire que les quatre biotopes se ressemblent en ce qui concerne les variations du taux d'oxygène et de sulfates (même orientation et même écartement des vecteurs $\mathrm{oO}_{2}$ et $\mathrm{oSO}_{4}$ par rapport au centre de gravité des deux premiers axes sortis $\mathbf{M}_{1}$ et $\mathbf{M}_{2}$ ). Or, si nous comparons avec les données initiales et les graphiques saisonniers correspondants, il y a similitude de variation pour l'oxygène et les sulfates des quatre biotopes.

Cependant, des divergences profondes apparaissent pour la silice, le gaz carbonique et le fer.

3.2.1. - La silice. - Nous pouvons rapprocher les stations 1 et 3 d'une part, 2 et 4 d'autre part. Les points $\mathrm{SiO}_{2}$ sont à l'extérieur du figuré mensuel pour 2 et 3 ; ils sont, par contre, à l'intérieur, donc très rapprochés du centre de gravité des deux premiers axes $M_{1}$ et $M_{2}$. Cependant, il est difficile de retrouver ces similitudes et ces divergences à l'examen des différents graphiques initiaux.

Nous pouvons peut-être expliquer ces groupements de stations par le fait que 1 et 3 sont des biotopes de bordure, peu profonds et riches en végétaux. Par contre, 2 et 4 sont des milieux de pleine eau, très profonds et pauvres en plantes aquatiques.

3.2.2. - Le fer et le gaz carbonique. - Pour les stations 1, 2 et 3 , les points $\mathrm{Fe}$ et $\mathrm{CO}_{2}$ sont très rapprochés. Cependant, le biotope 4 semble s'écarter des trois autres. Nous constatons, d'ailleurs, que la représentation graphique des variations saisonnières en $\mathrm{CO}_{2}$ et $\mathrm{Fe}$ est, pour le biotope 4, différente de celle que nous pouvons observer pour les stations 1,2 et 3 . Or, notre mare isolée est le seul milieu clos dix mois sur douze, pauvre en végétaux, ne recevant aucun apport extérieur, mis à part les excrétats des nombreux * troupeaux qui viennent s'abreuver durant la période estivale (juin à septembre). Nous retrouverons plus loin cette originalité du 

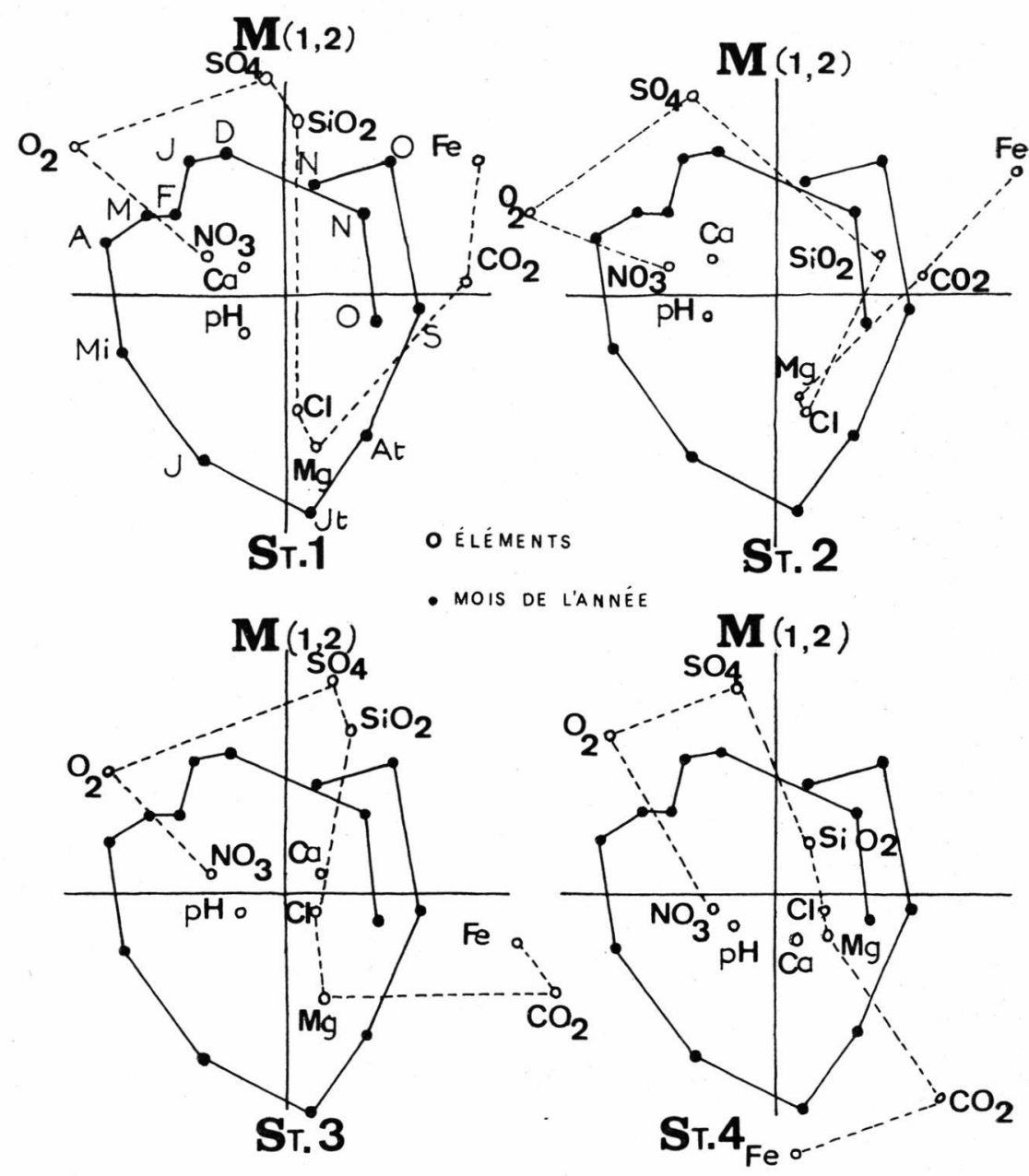

A NAGLO PAR Station - FIG. 4

biotope 4 dans les analyses partielles à propos du gaz carbonique et nous tenterons de l'expliquer.

3.2.3. - Les chlorures, le magnésium et la silice. - Nous pouvons noter une similitude de variation entre les chlorures, le magnésium, la silice. En effet, pour nos quatre biotopes, si les points $\mathrm{Cl}$ et $\mathrm{Mg}$ sont éloignés l'un de l'autre, ils le sont également des points $\mathrm{SiO}_{2}$. - Par contre, si les points $\mathrm{Cl}$ et $\mathbf{M g}$ sont très rapprochés, ils sont aussi très proches des points $\mathrm{SiO}_{2}$. Or, nous constatons une similitude de variation de ces trois éléments dissous surtout en période esti- 
vale et pré-automnale. Les taux de silice, de magnésium et de chlorures atteignent ensemble leurs plus hautes valeurs. Ces augmentations parallèles peuvent, d'ailleurs, être la conséquence directe des fortes évaporations estivales (rareté des précipitations atmosphériques et longues périodes d'ensoleillement).

\section{3. - Les analyses PaRtielles ( $f i g .5$ ).}

3.3.1. - Antagonisme $\mathrm{CO}_{2}-\mathrm{O}_{2}$ et $\mathrm{Fe}-\mathrm{O}_{2}$. - Nous retrouvons dans l'analyse partielle (biotope par biotope) les divergences observées dans les variations de l'oxygène et du gaz carbonique. Les points $\mathrm{O}_{2}$ et $\mathrm{CO}_{2}$, pour nos quatre stations, évoluent en sens inverse : sens des aiguilles d'une montre pour l'oxygène, sens trigonométrique pour $\mathrm{CO}_{2}$ et $\mathrm{Fe}$. Or, ces observations correspondent bien, nous

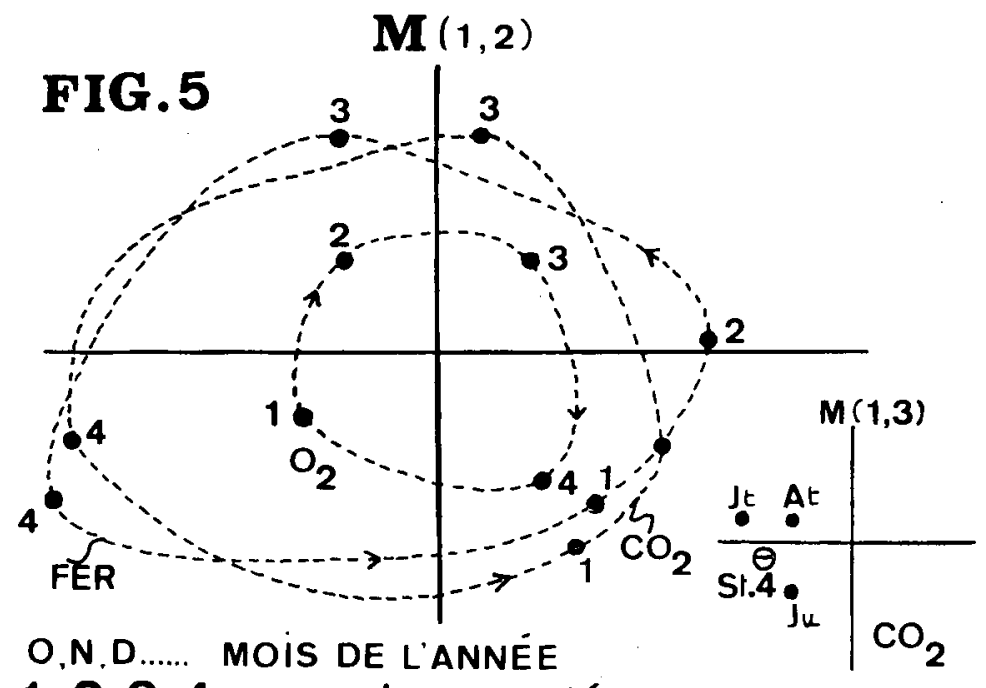

1,2,3,4: stations de. L'ÉTANG
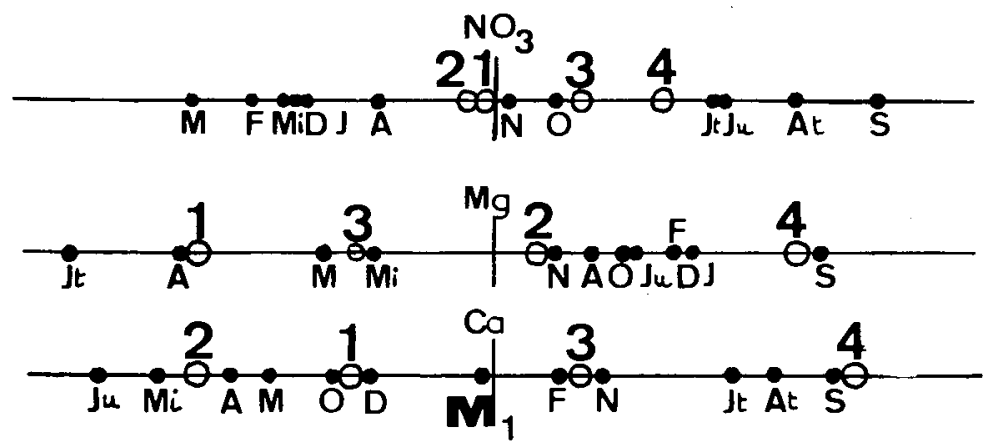

ANALYSES PARTIELLES 
venons de le voir plus haut, à la représentation graphique des données saisonnières.

3.3.2. - Originalité du biotope 4. - La station 4 s'individualise très nettement en ce qui concerne les nitrates, le calcium, le magnésium et le gaz carbonique. Nous pouvons, d'ailleurs, le vérifier si nous consultons les graphiques initiaux. Cette originalité est très nette durant les mois de juin, juillet et août. Sur le premier axe sorti $\left(\mathbf{M}_{1}\right)$ le point $\mathrm{St}_{4}$ s'écarte des trois autres pour $\mathrm{NO}_{3}, \mathrm{Ca}$ et $\mathrm{Mg}$. Nous pouvons ajouter que sur les axes $M_{1}$ et $M_{3}$ le biotope 4 se retrouve encore seul. En outre, nous notons que pour la station 4, les points $\mathrm{NO}_{3}, \mathrm{Ca}, \mathrm{CO}_{2}$ sont groupés avec les points représentant les mois de juin, juillet et août. Nous allons tenter d'expliquer l'originalité de la station 4. De nombreux troupeaux viennent régulièrement s'abreuver en période estivale, dans cette station. Les excrétats organiques rejetés dans le milieu clos et confiné sont importants. Or, l'urine des mammifères est riche en calcium et en azote (urée et acide urique). Nous pouvons alors penser que sous l'action de bactéries aérobies telles que les Bacillus, Proteus et Pseudomonas, l'urée est hydrolysée en carbonate d'ammonium puis en ammoniaque et gaz carbonique. Nous constatons, d'ailleurs, que la teneur en $\mathbf{N} \mathbf{N H}_{3}$ est la plus élevée dans cette station 4 . Il faut signaler aussi que cet azote ammoniacal peut facilement se convertir en nitrates $\left(\mathrm{NO}_{3}{ }^{-}\right)$sous l'influence de la flore bactérienne nitrificatrice; ceci confirmant l'importance de l'ion $\mathrm{NO}_{3}{ }^{-}$dans notre mare isolée.

\section{4. - Validité de la méthode.}

Ce traitement mathématique mis à l'épreuve pour la première fois en hydrobiologie mérite beaucoup d'attention et d'intérêt. En effet, il permet comme nous venons de le voir, de vérifier les similitudes et les divergences de variations saisonnières des éléments dissous de l'eau, d'une part dans un même biotope, d'autre part entre plusieurs stations.

\section{5. - Conclusions.}

Nous constatons tout d'abord que certains éléments comme l'oxygène, le gaz carbonique libre, la silice, les sulfates ont un cycle annuel de variation bien net, comportant un pic maximum et un pic minimum très importants. Cependant, pour la majorité des facteurs chimiques étudiés, le cycle saisonnier n'existe pratiquement pas; l'amplitude de variation de ces éléments est faible. C'est le cas des nitrates, des chlorures, des phosphates solubles, du magnésium, du calcium, enfin du $\mathrm{pH}$. 


\section{V. - CONCLUSIONS GENERALES}

L'étude des propriétés physico-chimiques des eaux nous a révélé que les milieux peuvent être classés dans le groupe des masses aquatiques «Oligotrophes », peu minéralisées, tamponnées essentiellement par du gaz carbonique et des bicarbonates, non par des phosphates comme dans les eaux du type Eutrophe. Nous avons retrouvé les variations saisonnières « classiques 》de chaque élément. Il était cependant intéressant de connaitre et d'expliquer les similitudes, les différences de variation entre plusieurs facteurs dans une même station. D'autre part, pouvait-on trouver des divergences d'un biotope à l'autre dans les fluctuations saisonnières des facteurs physico-chimiques de l'environnement? Il était à ce sujet très difficile de manœuvrer toutes les données de l'analyse. Aussi, avons-nous fait appel à un traitement mathématique des résultats obtenus.

Ainsi, nous avons représenté simultanément dans un espace à faibles dimensions l'ensemble des stations et des facteurs de l'environnement tout en respectant les valeurs initiales. Les résultats ont été encourageants car ils concordent dans leur ensemble aux graphiques initiaux représentatifs de l'évolution saisonnière des sels dissous de l'eau. Cette méthode nous aura aussi permis de voir que peu d'éléments ont un cycle annuel bien net, l'amplitude de variation de la plupart d'entre eux étant faible. Enfin ce traitement a montré que dans une masse aquatique donnée, des stations très proches peuvent évoluer différemment; tout paraissant dépendre de la profondeur, de la circulation des eaux, de la richesse en groupements végétaux, enfin des facteurs anthropo- et anthropo-zoobiotiques (milieux souillés ou perturbés par l'homme et les animaux).

\section{RÉSUMÉ}

L'auteur rappelle le but de ses recherches: préciser l'intervention du milieu environnant sur la composition et la dynamique saisonnière des populations algales d'un étang. Cette première partic est consacrée à la définition du milieu naturel choisi (étang de Planche-Roger). En effet, si l'on veut comprendre l'influence des facteurs du milieu sur la croissance des algues en place, il est nécessaire de décrire les variations de ces facteurs au cours d'un cycle complet de végétation.

L'étude des propriétés physico-chimiques des eaux montre tout d'abord que l'étang peut être classé dans le groupe des masses aquatiques «Oligotrophes», peu minéralisées, tamponnées essentiellement par du gaz carbonique et des bicarbonates. D'autre part on retrouve les variations saisonnières classiques pour chaque élément dans toutes les stations de l'étang.

Il était cependant très difficile de manœuvrer toutes les données de l'analyse. A cet effet l'auteur représente simultanément dans un espace 
à faible dimension l'ensemble des stations choisies et des facteurs de l'environnement en respectant au mieux les données initiales. Ce traitement mathématique des "Correspondances» permet de retrouver les valeurs saisonnières fournies par l'analyse (par exemple : l'antagonisme entre l'oxygène d'une part, le gaz carbonique et le fer d'autre part). Enfin ce procédé montre bien que dans un étang donné, des stations très proches peuvent évoluer différemment; tout paraissant dépendre de la profondeur, de la circulation des eaux, de la richesse en groupements végétaux, enfin des facteurs anthropo- et anthropozoobiotique (milieux souillés par l'homme ou les animaux).

\section{CONTRIBUTION TO THE BIOLOGY OF FRESHWATER ALGAE FROM A POND IN BRITTANY. I : THE NATURAL ENVIRONMENT}

The purpose of this research is to determine the effect of the surrounding environment on both the composition and seasonal dynamics of algal populations in a pond in Brittany. The first part is devoted to the definition of the selected environment (Planche-Roger's pond). Indeed, if one wants to understand the influence of environmental factors on the algal growth in a locality, it is necessary to describe changes in these factors in the course of a complete growth cycle.

The study of physiochemical properties of the water shows at once that the pond may be classed among aquatic bodies called "Oligotrophs", being a little mineralised but chiefly buffered on account of carbonic gas and bicarbonates. In the other part, classical seasonal variations are found for each element at all stations of the pond.

However it was very difficult to handle all the data of the analysis. To this purpose the author depicts simultaneously in a narrow space the whole group of the selected stations and environmental factors by respecting as well as possible the initial data. This mathematical manner of using "Correspondences" allows us to find out the seasonal values supplied through the analysis (e.g. antagonism between oxygen on the one hand and carbonic gas and iron on the other). Finally this process truly shows that in a given pond, very close stations may be working in a different way; everything seems to depend on depth, water movement, richness about clusters of aquatic plants and finally on both anthropo- and anthropozoobiotic factors (environment spoiled by man or animals).

\section{BEITRAG ZUR BIOLOGIE DER SUSSWASSERALGEN GINES TEICHES IN DER BRETAGNE I : DAS NATURLICHE MILEU}

Der Verfasser betont das Ziel seiner Forschungen :

Die genauere Bestimmung der Intervention des umgebenden Milieus auf die Zusammensetzung und die jahreszeitliche Dynamik der Algenpopulationen eines Teiches.

Dieser erste Teil ist der Definition des ausgesuchten natürlichen Milieus (étang de Planche-Roger) gewidmet. Wenn man den Einfluss der Milieufaktoren auf das Wachstum der Algen an Ort und Stelle verstehen will, kann man nicht umhin die Veränderungen dieser Faktoren im Laufe eines kompleten Vegetationszyklusses zu beschreiben.

Das Studium der physico-chemischen Eigenschaften des Gewässers zeigt zunächst dass der Teich in die Klasse der "oligotrophen", wenig 
mineralisierten und hauptsächlich von Kohlensäure und Bikarbonaten gepufferten Wassermassen gestellt werden kann. Weiterhin findet man in allen Stationen des Teiches für jeden Bestandteil die klassischen jahreszeitlichen Schwankungen.

Es war jedoch sehr schwierig, alle Analysenergebnisse zu handhaben. Zu diesem Zweck stellt der Verfasser gleichzeitig und in kleinem Masstab die Gesamtheit der gewählen Beobachtungsstationem mit den entsprechenden Milieufaktoren dar, wobei die ursprünglichen Gegebenheiten zum bestmöglichsten respektiert wurden.

Diese mathematische Behandlung der "Correspondenzen" führt auf die durch Analyse gefundenen jahreszeitlichen Messwerte zurück. (Zum Beispiel : Antagonismus zwischen Sauerstoff einerseits und Kohlensäure und Eisen andererseits.) Dieser Vorgehen zeigt klar dass sehr nahe gelegene Stationen in ein und demselben Teich verschiedene Entwicklungen durchlaufen können; alles scheint von der Tiefe, der Strömungen, der Verteilung des pflanzlichen Reichtums und nicht zuletzt von den anthropo- und anthropozoobiotischen Faktoren (von Mensch und Tier verschmutztes Milieu) abzuhängen. 


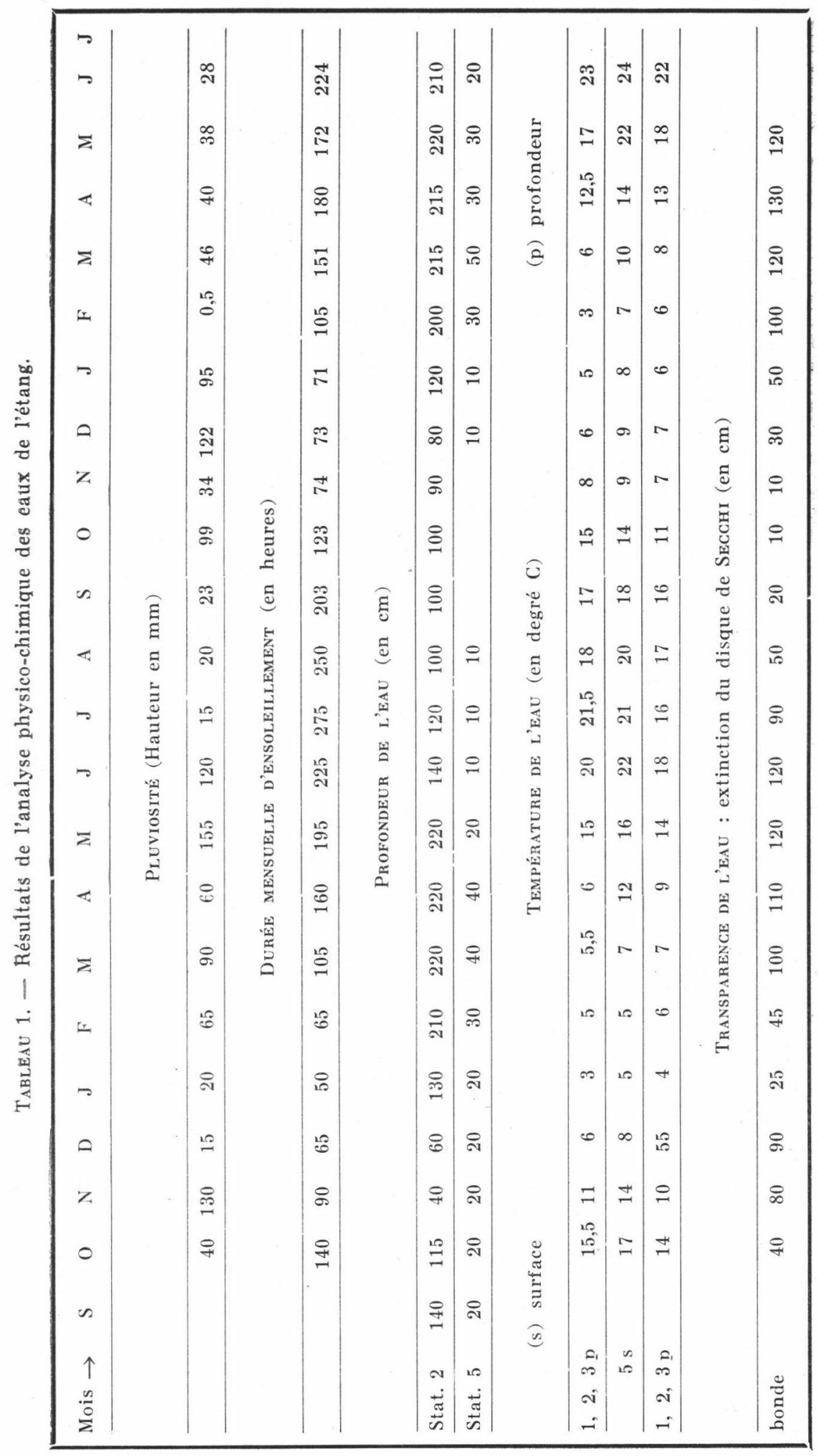




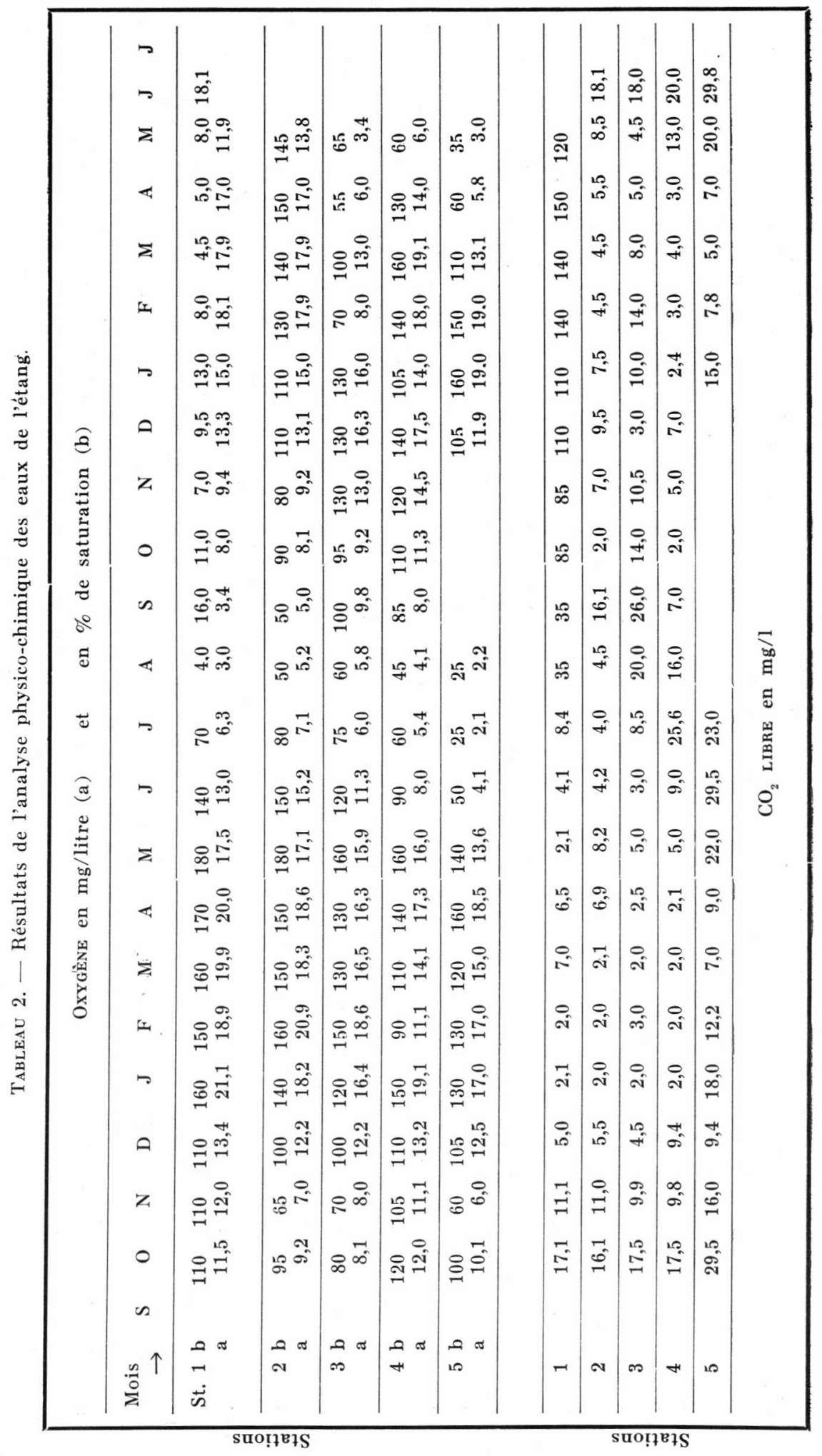




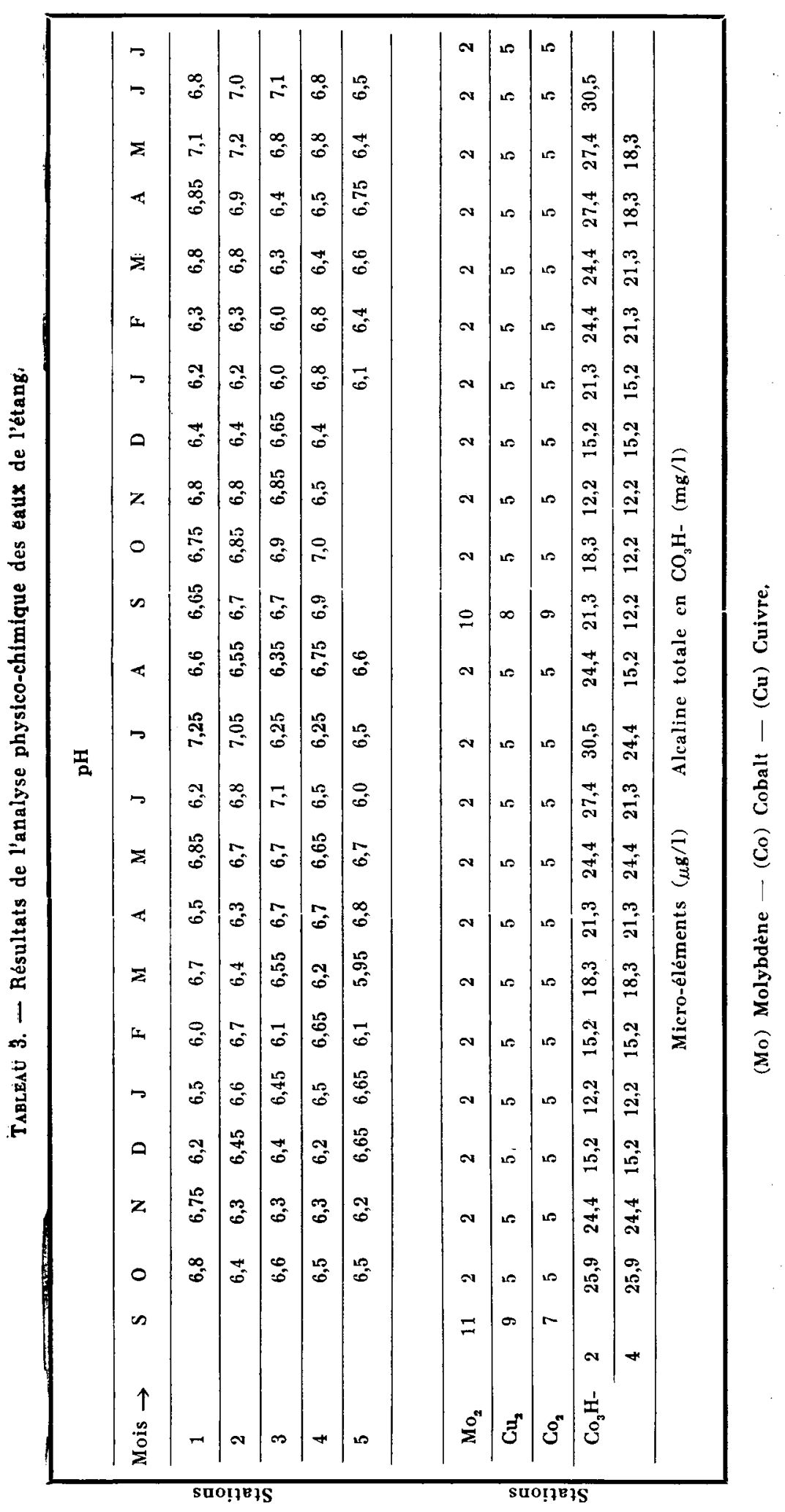




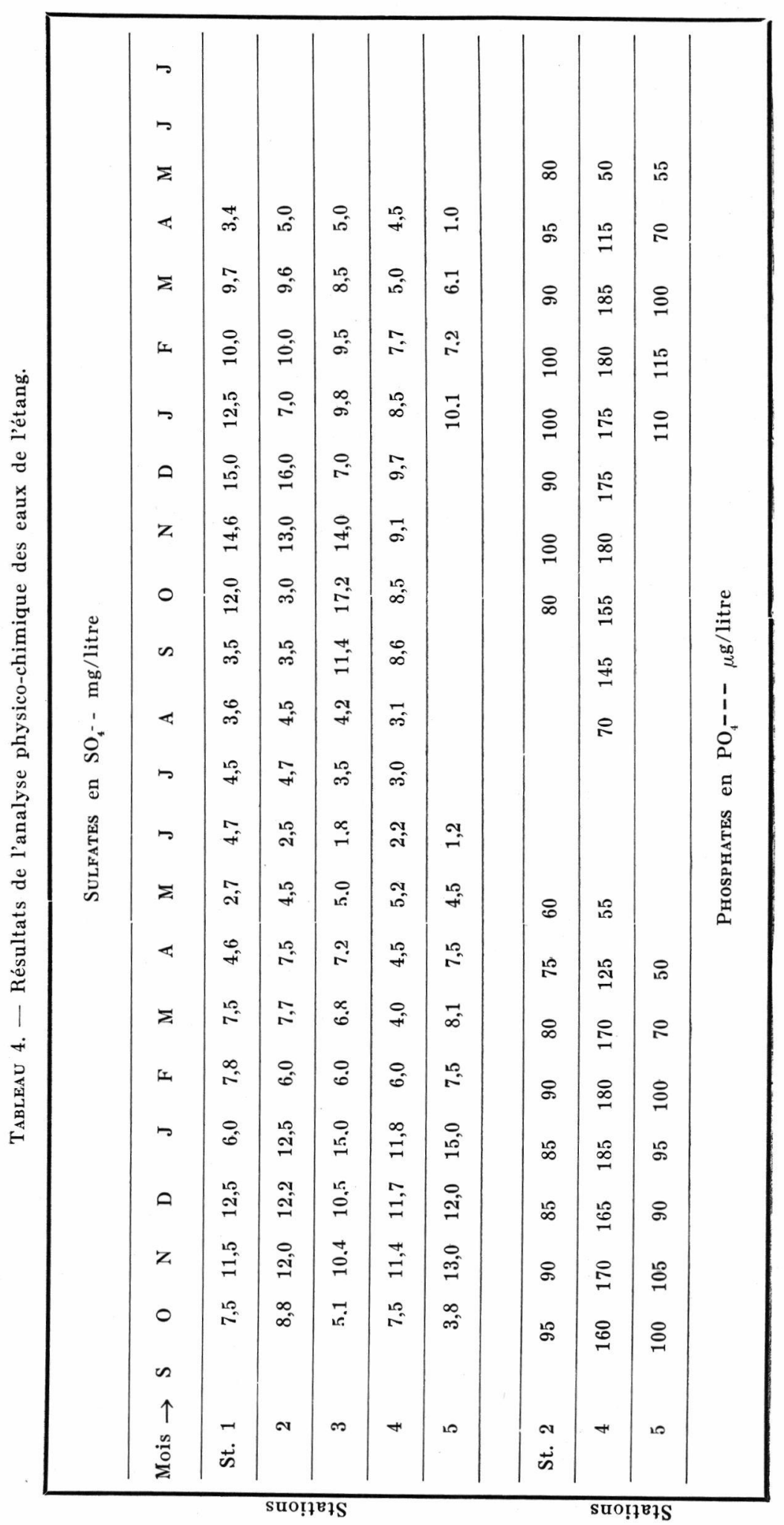




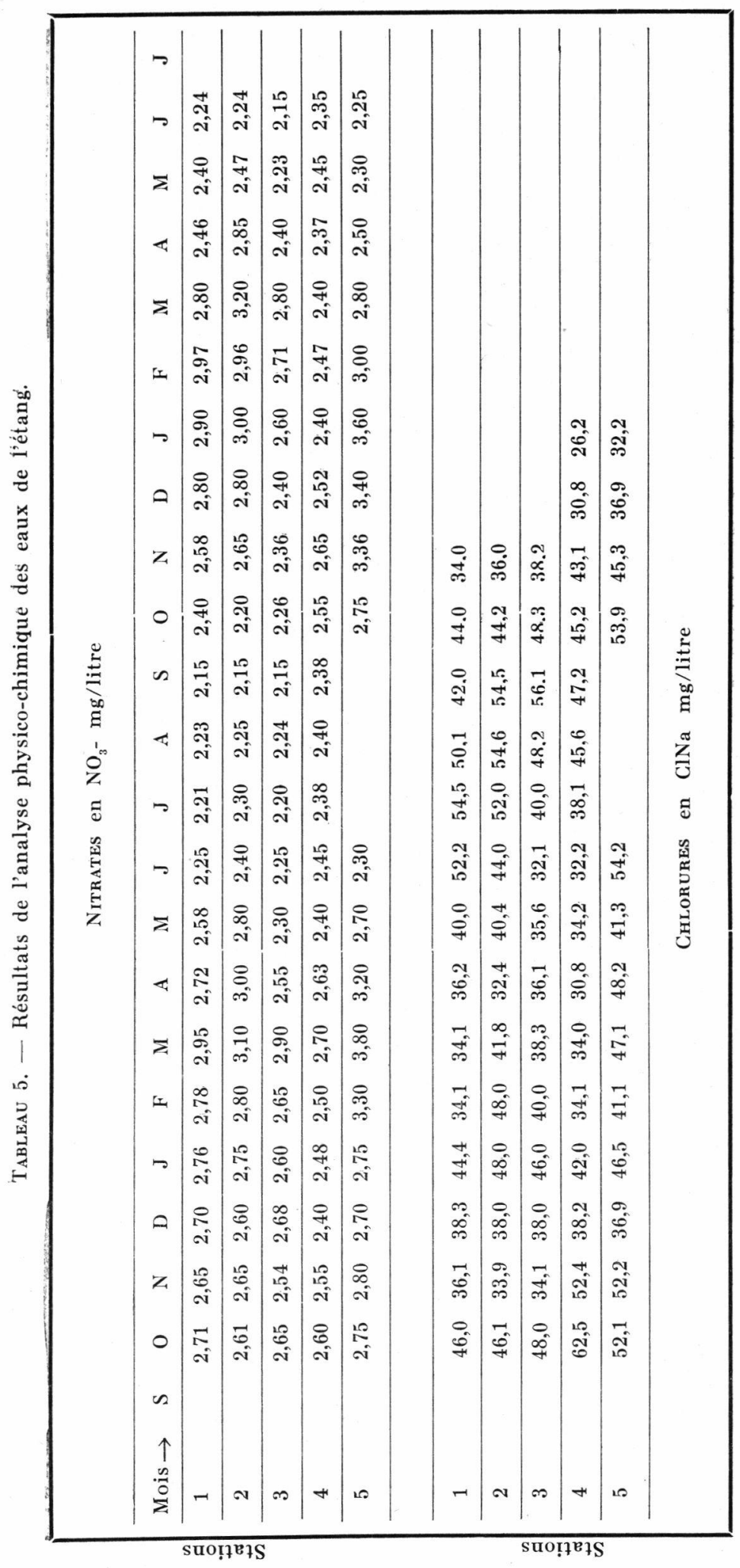


Tableau 6. - Résultats de l'analyse physico-chimique des eaux de l'étang.

Azote ammoniacal Libre

\begin{tabular}{|ccccccccccccc|}
\hline $\begin{array}{c}\text { Mois } \\
\rightarrow\end{array}$ & J & J & A & S & O & N & D & J & F & M & A \\
\hline St. & 1 & 0,20 & 0,31 & 0,38 & 0,35 & 0,25 & 0,12 & 0,10 & 0,12 & 0,08 & 0,13 & 0,20 \\
\hline St. & 2 & 0,12 & 0,22 & 0,31 & 0,45 & 0,50 & 0,20 & 0,09 & 0,07 & 0,09 & 0,07 & 0,11 \\
\hline St. & 3 & 0,07 & 0,15 & 0,30 & 0,38 & 0,32 & 0,20 & 0,05 & 0,07 & 0,07 & 0,05 & 0,07 \\
\hline St. & 4 & 0,22 & 0,43 & 0,80 & 0,90 & 0,75 & 0,50 & 0,38 & 0,18 & 0,12 & 0,15 & 0,22 \\
\hline
\end{tabular}

Azote ammoniacal albuminoide

\begin{tabular}{|ccccccccccccc|}
\hline $\begin{array}{c}\text { Mois } \\
\rightarrow\end{array}$ & J & J & A & S & O & N & D & J & F & M & A \\
\hline St. & 1 & 0,12 & 0,30 & 0,40 & 0,32 & 0,15 & 0,05 & 0,02 & 0,01 & 0,02 & 0,07 & 0,10 \\
\hline St. & 2 & 0,07 & 0,12 & 0,25 & 0,34 & 0,39 & 0,15 & 0,05 & 0,02 & 0,05 & 0,05 & 0,03 \\
\hline St. & 3 & 0,02 & 0,11 & 0,22 & 0,30 & 0,35 & 0,15 & 0,02 & 0,05 & 0,04 & 0,03 & 0,03 \\
\hline St. & 4 & 0,14 & 0,15 & 0,23 & 0,24 & 0,22 & 0,10 & 0,05 & 0,04 & 0,05 & 0,05 & 0,08 \\
\hline
\end{tabular}

Les valeurs indiquées sont exprimées en $\mathrm{mg} / \mathrm{l}$ de $\mathrm{N} . \mathrm{NH}_{\mathbf{z}}$. 


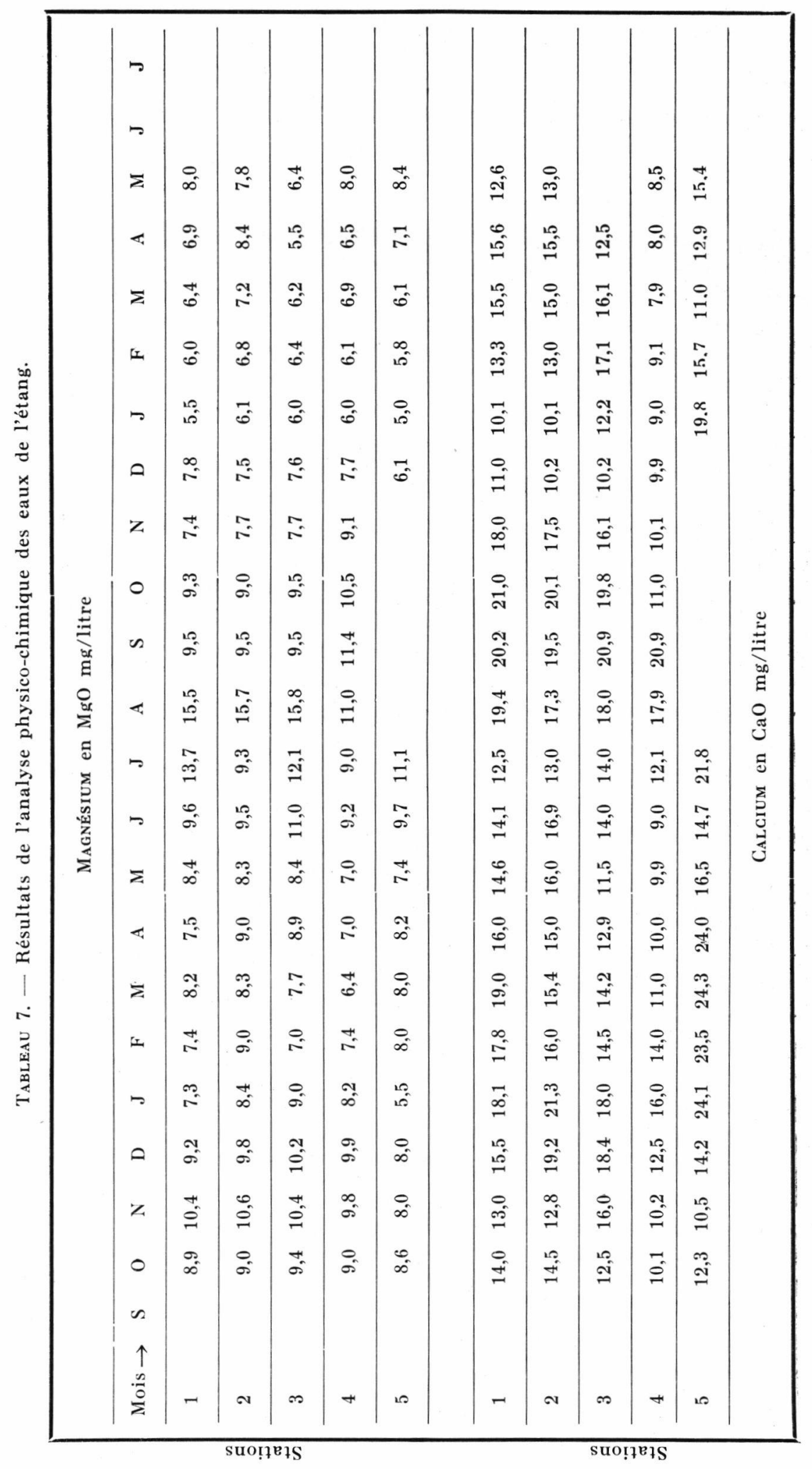




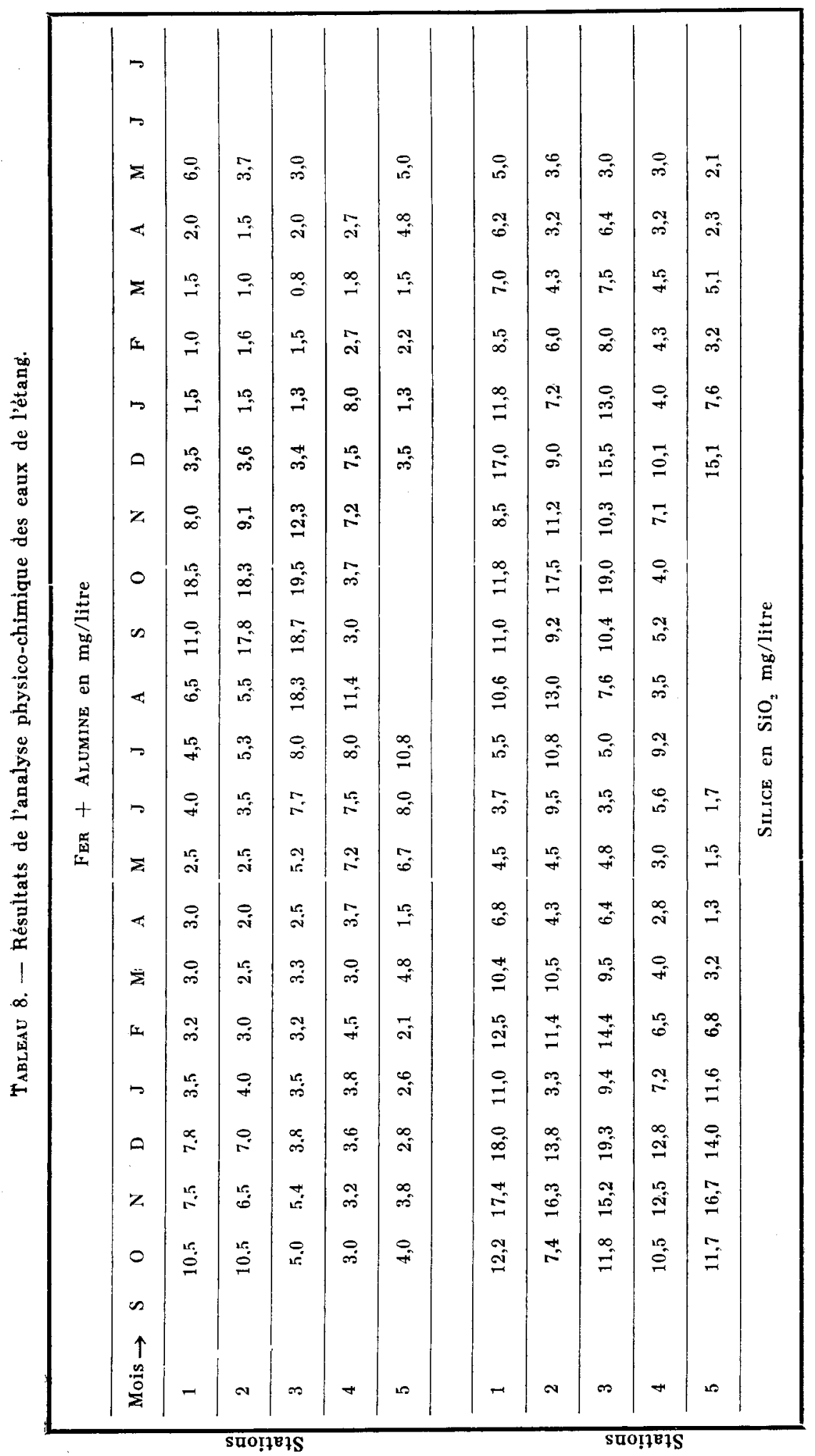




\section{TRAVAUX cITES}

APHA. 1965. Standard methods for the examination of water and waste water. Twelfth edition, New-York : $769 \mathrm{p}$.

Bourel (M.), Lenoir (P.), Kerbaol (M.). 1968. - Analyse des Correspondances; application aux données médicales. Path. Biol., 16, nx 15-16/17-18 : 715-721.

Burriel (F.), Gallego (R.). 1953. - Dosage colorimétrique du cobalt par le sel Nitroso R; une méthode proposée et ses applications. Real. Soc. Espana Fis. Yqum 47 B., 587-590.

Capelle (R.). 1960. -- Dosage colorimétrique du cuivre au moyen de la bis cyclohexanone oxalydihydrazide. Chim. Anal., $42: 69-78$ et 127-135.

Chandler (D. C.). 1942. - Limnological studies of Western lake Erié; 2 : Light penetration and its relation to turbidity. Ecology, 23 : 41-52.

Dickman (S. R.). 1946. - Colorimetric determination of phosphorus. Ind. Eng. Chem. Anal. Ed., 12 : 665-668.

Dirscheri. (A.), Erne (F.). 1960. - Anwendung der Polbenverbrennung auf die Mikrobestimmung der Phosphore in organischen substanzen. Mikrochim. Acta, 5, $\mathrm{n}^{\circ} 6$ : 779-788.

Ducet (G.), Van de Walle (G.). 1959. - Nouvelles techniques du dosage du phosphore. Ann. Phys. Vég., 2 : 199-205.

Dussart (B.). 1966. - Etude des eaux continentales. Paris, GauthiersVillars : $677 \mathrm{p}$.

Gedroits (K. K.). 1963. - Chemical analysis of soils. Israel Program for Scientific Translations, Jerusalem, $602 \mathrm{p}$.

Ging (N. S.). 1956. - Méthode d'extraction pour le dosage colorimétrique du phosphore pour des quantités de l'ordre du microgramme. Anal. Chem., 28 : 1850-1853.

Goldman (C. R.). 1960. - Molybdenum as a factor limiting primary production in Castle lake, California. Science U.S.A., 132, $\mathrm{n}^{\circ} 3433$ : 1016-1017.

Harvey (H. W.). 1953. -- Microdosage du phosphore dans le matérieI biologique. Analyst., 78 : 110-114.

HASIER (A. D.). 1947. - Eutrophisation of lakes by domestic drainage. Ecology, 28 : 383-395.

Heron (J.). 1961. - The seasonal variation of phosphate, silicate and nitrates in waters of the English lake District. Limnol. Oceanogr., 6 : 338-346.

Hirata (A. A.), Appieman (D.). 1959. - Microdetermination of phosphorus in the range of 1 to $10 \mu \mathrm{g} / \mathrm{ml}$. Analyst. Chem., 31 (12) : 2097-2099.

HöHNE (E.). 1963. - Biologische, chemische, physikalische Untersuchungen an den Trinkwasser Teichen der Stadt Freiberg (Sachsen). Wiggens. Zeits. der Karl Marx Univ. Leipzig, 1 : 193-231.

Horwith (W.). 1960. - Official methods of analysis of the association of official agricultural chemists. Association of Official Agricultural Chemists : 832 p.

KAILA (A.). 1954. - Iron as a disturbing factor in the determination of phosphate by the molybdenum blue method. Jour. Sc. Agr. Soc. Finland, 26 : 159-168. 
Lenolr (M.). 1958. - Les ceintures de végétation des étangs de la région de Paimpont (Ille-et-Vilaine). Bull. Soc. sci. Bretagne : 97-133.

Nakamura (N.). 1954. - A new device to mesure the transparency of water in the fish culture pond. Bull. Jap. Soc. sci. fish., 19 (11) : 1096-1099.

Pinta (M.). 1962. - Recherche et dosage des éléments traces. Dunod, Paris : 726 p.

Profr (G.). 1964. - Bestimmung von Gesauertphosphor in Wasser und Abwasser als molybdo-vanado-phosphosaure. Limnologica, 11 (4) : 407-409.

QuinhaM (K. P.), De SESA (M. A.). 1955. - Dosage spectrophotométrique du phosphore à l'état d'acide phosphomolybdovanadique. Annal. Chem., 27 : 1626-1629.

Rawson (D. S.). 1951. - The total mineral content of lake waters. Eco$\log , 32: 669-672$.

Rodнe (W.). 1949. - The ionic composition of lake waters. Verh. Intern. Verein. Limnol., $10: 377-386$.

Rodier (J.). 1966. - L'analyse physicochimique de l'eau. Dunod, Paris : $412 \mathrm{p}$.

SAvouré (B.), Villeret (S.). 1964. - Contribution à l'étude hydrobiologique de la Bretagne. I : Florule algale de quelques étangs du département de l'Ille-et-Vilaine (à l'exception des Diatomées). Bull. Soc. sci. Bretagne, 39 : 233-241.

Sıvouré (B.), LE CoHU (R.). 1966. - Contribution à l'étude hydrobiologique de la Bretagne. II : Florule algale de quelques étangs du département des Côtes-du-Nord. 91 Congrès Soc. Sav. Rennes, 3 : 19-34.

Savouré (B.), Le Cohu (R.), Bertru (G.). 1967. - Contribution à l'étude hydrobiologique de la Bretagne. III : Quelques étangs de la LoireAtlantique et du Maine-et-Loire (région de Pouancé). Bull. Soc. sci, Bretagne, 43 : 259-269.

Sıvouré (B.). 1968. - Contribution à l'étude hydrobiologique de la Bretagne. IV : Quelques étangs du département de l'Ille-et-Vilaine. Bull. Soc. sci. Bretagne, $43: 43-48$.

Sivouré (B.). 1969. - Contribution à l'étude hydrobiologique de la Bretagne. $\mathrm{V}$ : Quelques étangs du département d'Ille-ct-Vilaine. Bull. Soc. sci. Bretagne, 44 : 25-31.

Silvey (W. D.). 1961. - Concentration method for the spectrochemical determination of minor elements in waters. Geol. Surv. Water Supply Paper, 1540 B : 11-22.

Talvitie (N. A.), Pérez (E.), Illustre (D. P.). 1962. - Spectrophotometric determination of phosphorus as molybdovanadophosphoric acid. Annal. Chem., 34 (7) : 866-867.

VAN Beneden (G.). 1957. - Réactifs en poudre pour la recherche et le dosage des nitrates et des nitrites. Bull. Centre Belge Eaux, 38 : 250-259.

VirLeret (S.). 1953. - Contribution à la biologie des algues des tourbières à sphaignes. Bull. Soc, sci. Bretagne, f. hors-série, $29: 246$ p.

Villeret (S.), SavourÉ (B.). 1957. - $\mathrm{pH}$, pouvoir tampon et typologie des eaux douces. C. R. Soc. Biogéogr., $305: 37-43$.

Villeret (S.). 1960. - Note de Limnologie : Un appareil simple et peu coûteux pour le dosage de l'oxygène dans les eaux. Bull. Soc. sci. Bretagne, 35 : 169-172.

Williams (L. G.), Mount (D. I.). 1965. Influence of $\mathrm{Zn}$ on periphytic communities. Amer. Jour. Bot., $52(1)$ : 26-34. 
Wooley (J. T.), Hicks (G. P.), Hageman (R. H.). 1960. - Plant analysis; rapid determination of nitrat and nitrit in plant materials. Jour. Agric. Food Chem., y (6) : 481-482.

\section{(Laboratoire d'Hydrobiologie de la Faculté des Sciences de Rennes, avenue du Général-Leclerc, 35-Rennes.)}

